\title{
Decision Maker Approaches for Cooperative Spectrum Sensing: Participate or Not Participate in Sensing?
}

\author{
Angela Sara Cacciapuoti, Member, IEEE, Marcello Caleffi, Member, IEEE, \\ Luigi Paura, Member, IEEE, and Roberto Savoia
}

\begin{abstract}
Cooperative spectrum sensing techniques are mainly based on two different decision approaches, according to the role of the decision maker: i) in the Combining Decision approach, the decision maker combines the sensing information collected from its cooperators, without participating in the sensing of the monitored band; ii) in the Sensing \& Combining Decision approach, the decision maker combines both the sensing information of its cooperators and its own local sensing information. The choice of the decision approach deeply affects the performance of any cooperative spectrum sensing technique. However, the key issue of choosing the decision approach that guarantees the higher detection accuracy independently of the underlying cooperative sensing architecture is still an open problem. For this, in this paper, the criteria for an effective decision-approach selection are analytically derived with the object of maximizing the detection accuracy in presence of realistic channel propagation effects. Specifically, through a theoretical analysis, it is proven that the detection accuracy exhibits a threshold behavior as a function of the adopted decision approach. Closed-form expressions of such a threshold are analytically derived and practical insights for the decision approach choice are provided. Finally, the theoretical analysis is validated through simulations.
\end{abstract}

Index Terms-Cognitive radio, spectrum sensing, cooperative, detection accuracy.

\section{INTRODUCTION}

$\mathbf{S}$ PECTRUM sensing is a key functionality of the Cognitive Radio (CR) paradigm [1]. Through spectrum sensing, unlicensed users, referred to as Secondary Users (SUs), can identify and dynamically exploit the spectrum holes, i.e., the portions of the radio spectrum that are temporarily not occupied by the licensed users, referred to as Primary Users (PUs). The wireless-channel impairments, such as multipath fading and shadowing, affect the sensing reliability. Thus, recently, cooperative spectrum sensing has been proposed as an effective way to improve the sensing performance by exploiting the spatial diversity among the SUs. By cooperation, SUs share their local sensing information through

Manuscript received August 1, 2012; revised November 26, 2012; accepted February 4, 2013. The associate editor coordinating the review of this paper and approving it for publication was $\mathrm{S}$. Wei.

The authors are with the Department of Electrical Engineering and Information Technology, University of Naples Federico II, Italy (e-mail: \{angelasara.cacciapuoti, marcello.caleffi, paura, roberto.savoia\}@unina.it).

This work was supported by the Italian government under Grants PON0100744 "DRIVE-IN2: DRIVEr monitoring: technologies, methodologies, and IN-vehicle INnovative systems for a safe and ecocompatible driving,' PON0101936 "HABITAT: HarBour traffIc opTimizAtion sysTem," and PON0103096 "MC3-Care: Mobile Continuos Connected Comprehensive-Care."

Digital Object Identifier 10.1109/TWC.2013.031813.121112 links, referred to as reporting channels (RCs), and a decision maker combines these sensing statistics to take the cooperative decision about the presence of the PU [2]-[7].

The cooperative sensing techniques available in literature are mainly based on two different decision approaches, according to the role of the decision maker. In the first approach, referred to as Combining Decision (CD), the role of the decision maker is only to combine the sensing information collected from its cooperators, without participating in the sensing of the monitored band. Differently, in the second approach, referred to as Sensing \& Combining Decision (SCD), the decision maker combines not only the sensing information of its cooperators, but also its own local sensing information.

Traditionally, the choice between the CD and SCD approaches is based on the adopted cooperative sensing architecture [3]. More in detail, the CD approach is adopted in centralized cooperative sensing architectures ${ }^{1}$, whereas the SCD approach is adopted in distributed cooperative sensing architectures ${ }^{2}$. This choice is mainly historical, due to the application of the classical centralized versus distributed paradigm in CR scenarios. Nevertheless, in the appealing context of the CR Ad-Hoc Networks (CRAHNs) [8], the aforementioned choice to associate the $\mathrm{CD}$ and the SCD approaches to centralized and distributed cooperative sensing architectures, respectively, is not mandatory. Hence, any arbitrary cooperative sensing technique can be implemented according to either the $\mathrm{CD}$ or the SCD approach, regardless of the underlying cooperative sensing architecture.

As we prove through the paper, the adoption of the $\mathrm{CD}$ or the SCD approach deeply affects the performance of the considered cooperative sensing technique, in terms of detection accuracy and, consequently, in terms of PU interference avoidance [3]. However, the key issue of choosing the decision approach that guarantees the higher detection accuracy independently of the underlying cooperative sensing architecture is still an open problem.

For this reason, in this paper, we derive the criteria for an effective decision-approach selection. To the best of our

\footnotetext{
${ }^{1}$ Centralized cooperative sensing architecture denotes a cooperative sensing based on a central entity, referred to as Fusion Center (FC), that handles the entire cooperative process. In infrastructure-based networks, the CR basestation is naturally the FC, whereas in CRAHNs any SU can act as FC [3].

${ }^{2}$ Distributed cooperative sensing architecture denotes a cooperative sensing that does not rely on a central entity that handles the cooperative process, but the task is distributed among the SUs acting as decision makers.
} 


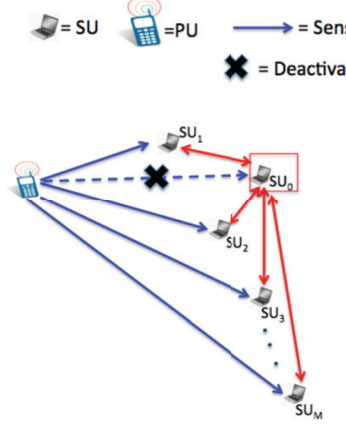

(a)

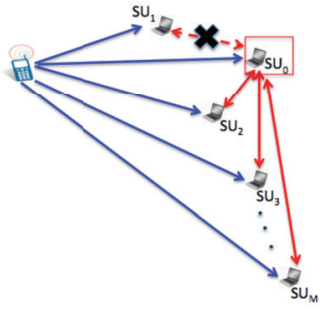

(b)
Fig. 1. (a) CD approach; (b) SCD approach.

knowledge, this is the first work that addresses this issue.

Specifically, we prove that the detection accuracy of a cooperative spectrum sensing technique exhibits a threshold behavior as a function of the adopted decision approach: it exists a threshold value that determines two different operating regions, depending on the sensing and reporting channel parameters. In one region, the higher detection accuracy is assured by implementing the cooperative sensing technique according to the $\mathrm{CD}$ approach, whereas in the other region, the higher detection accuracy is guaranteed by the SCD approach, regardless of the underlying cooperative sensing architecture and the adopted sensing technique ${ }^{3}$.

The rest of the paper is organized as follows. In Sec. II, we present the problem statement, along with the contributions of the paper. In Sec. III, we describe the system model. In Sec. IV we present intermediate results that will be used in Sec. V to prove the existence of the threshold behavior in the detection accuracy. We validate the analytical results by simulation in Sec. VI. In Sec. VII, we conclude the paper, and, finally, some proofs are demonstrated in the appendices.

\section{Problem Statement and Contributions}

In the following, we first present some definitions used through the paper, then we formulate the problem statement along with the contributions of the paper.

\section{A. Problem Statement}

Let us consider a typical cooperative sensing scenario in which $M$ SUs cooperate to take the decision about the PU presence on the monitored band. In the rest of the paper, we consider an equal number $M$ of cooperative SUs in both the $\mathrm{CD}$ and the SCD approaches for a fair comparison.

Definition 1. Combining Decision (CD) Approach. The decision maker combines, according to a certain criterion, only the local sensing data of its cooperators, without participating in the sensing of the monitored band, as depicted in Fig. 1-(a).

Remark 1. Definition 1 implies that all the $M$ sensing statistics are sent from the SU neighbors to the decision maker through the reporting channels, since the decision maker does

\footnotetext{
${ }^{3}$ In Sec. V, we prove that the adopted cooperative sensing technique influences only the value of the threshold, not its existence.
}

not participate in the sensing of the monitored band. Thus, in Fig. 1-(a), the sensing link between the decision maker and the arbitrary PU is denoted as deactivated.

Definition 2. Sensing \& Combining Decision (SCD) Approach. The decision maker combines, according to a certain criterion, both the sensing data received from its cooperators and its own local sensing data, as depicted in Fig. 1-(b).

Remark 2. Definition 2 implies that $(M-1)$ sensing statistics (rather than $M$ ) are sent from the SU neighbors to the decision maker through the reporting channels, since a sensing statistic is locally available at the decision maker. Thus, in Fig. 1(b), the reporting channel between the decision maker and the arbitrary $\mathrm{SU}_{1}$ is denoted as deactivated.

In Remarks 3 and 4 we discuss the applicability of the CD and SCD approaches in both centralized and distributed cooperative sensing architectures.

Remark 3. The CD is the traditional approach adopted in centralized cooperative sensing architectures. In fact, in such a case, the decision maker ( $\mathrm{SU}_{0}$ in Fig. 1-a), acts only as $\mathrm{FC}$ and the other SUs perform local sensing and report the results back to $\mathrm{SU}_{0}$ [3]. Differently, adopting the SCD approach in centralized cooperative sensing architectures constitutes a new hybrid solution, in which the decision maker $\mathrm{SU}_{0}$ not only acts as FC, but it also participates in the sensing of the band.

Remark 4. The SCD is the traditional approach adopted in distributed cooperative sensing architectures. In fact, in such a case, each SU exchanges its sensing information with its neighbors and then it combines its local data with the received sensing data for deciding on the PU presence ${ }^{4}$ [3]. Differently, adopting the $\mathrm{CD}$ approach in distributed cooperative sensing architectures constitutes a new hybrid solution, in which an arbitrary SU combines the received sensing data, without participating in the sensing of the monitored channel band.

Problem Definition:. To analytically single out the criteria establishing how to choose the decision approach that guarantees the higher detection accuracy independently of the underlying cooperative sensing architecture. From Definitions 1 and 2, this problem can be reformulated as to analytically single out the criteria establishing when it is more advantageous in terms of detection accuracy to use the local sensing statistic of the decision maker, by allowing it to participate in the sensing process, than to involve another SU in the cooperative process.

\section{B. Contributions}

In this paper, we solve the aforementioned decision approach problem through a theoretical analysis, by adopting

\footnotetext{
${ }^{4}$ Traditionally, in distributed cooperative sensing architectures, the SUs repeat the described process to converge to a unified decision [3]. In this paper, we focus on enhancing the reliability of the decision made by each SU by selecting the effective decision approach, since, clearly, this enhances in turn the reliability of the possible unified decision. Moreover, we observe that reaching a unified decision could be not practicable for two main reasons: $i$ ) in dynamic PU environments, the long decision delays induced by the iterative nature of the unified-decision techniques could not be tolerable; $i i$ ) if the RCs are not ideal, the underlying assumption of the unified-decision techniques, i.e., to have a reliable decision exchange among the SUs, is violated. Hence, in these scenarios, enhancing the reliability of the decision made by each SU is even more important.
} 
the realistic multipath frequency-selective channel model for the RCs. To guarantee generality to the proposed analysis, we consider three different cooperative sensing techniques, implemented according to both the decision approaches: the optimal Likelihood Ratio-Test (LRT) based on the NeymanPearson criterion [9] used as benchmark, and two techniques, based on a Linear (L) and Widely-Linear (WL) processing.

We observe that the CD design of a $L$ and a WL cooperative technique able to work in presence of temporal dispersive RCs has been recently proposed in literature [4], [10], as we discuss in Sec. IV. Differently, to the best of our knowledge, no technique designed according to the SCD approach able to work in presence of temporal dispersive RCs is available in literature. Motivated by this, to carry out the theoretical comparison between the two decision approaches, in this paper we also design both a $\mathrm{L}$ and WL cooperative techniques according to the SCD approach for dispersive RCs (see Sec. IV).

In a nutshell, the contribution of the paper is threefold: $i$ ) we design two cooperative techniques for the SCD approach, able to counteract the RC temporal dispersion; $i i$ ) we analytically derive the criteria for choosing the decision approach assuring the higher detection accuracy, independently of the underlying cooperative sensing architecture; iii) we analytically prove that the detection accuracy exhibits a threshold behavior as a function of the adopted decision approach, and we provide the closed-form expression of the threshold value.

\section{SySTEM MODEL}

Here $^{5}$, we first describe the system model at each SU, then the system model for both the CD and the SCD approaches, by accounting for temporal dispersive RCs.

\section{A. The Local Sensing Model}

We consider a CR network with $M$ cooperative SUs. The base-band received signal $x_{i}(k)$ at the $i$-th SU is [3], [4]:

$$
x_{i}(k)= \begin{cases}v_{i}(k) & \mathcal{H}_{0} \\ g_{i} s(k)+v_{i}(k) & \mathcal{H}_{1}\end{cases}
$$

where $s(k)$ is the PU's transmitted signal, $g_{i}$ is the complex channel coefficient that models the sensing channel (SC) between the PU and the $i$-th $\mathrm{SU}, v_{i}(k) \sim \mathcal{C N}\left(0, \sigma_{i}^{2}\right)$ is the complex zero-mean Additive White Gaussian Noise (AWGN), assumed circular (or proper) [12], i.e., $E\left[v_{i}(n) v_{i}(m)\right]=$ $0, \forall n, m \in Z$. Each SU adopts an energy detector as local

\footnotetext{
${ }^{5}$ Notations: $\mathcal{H}_{0}$ and $\mathcal{H}_{1}$ denote the hypotheses of absence and presence of the PU, respectively; the fields of complex and real numbers are denoted with $\mathbb{C}$ and $\mathbb{R}$; matrices [vectors] are denoted with upper case [lower case] boldface letters (e.g., $\mathbf{X}$ or $\mathbf{x}$ ); the field of $m \times n$ complex [real] matrices is denoted as $\mathbb{C}^{m \times n}\left[\mathbb{R}^{m \times n}\right]$, with $\mathbb{C}^{m}\left[\mathbb{R}^{m}\right]$ used as a shorthand for $\mathbb{C}^{m \times 1}\left[\mathbb{R}^{m \times 1}\right]$; the superscripts $*, T, H,-1$ and $\dagger$ denote the conjugate, the transpose, the Hermitian (conjugate transpose), the inverse of a matrix and the MoorePenrose inverse (generalized inverse) [11], respectively; the subscripts c and sc denote the $\mathrm{CD}$ and the SCD approaches, respectively; $\mathbf{I}_{m} \in \mathbb{R}^{m \times m}$, $\mathbf{0}_{m \times n} \in \mathbb{R}^{m \times n}$ and $\mathbf{1}_{m} \in \mathbb{R}^{m} \triangleq[1, \ldots, 1]^{T}$ denote the identity matrix, the null matrix and the unitary vector, respectively; $\operatorname{trace}(\mathbf{X})$ and $\operatorname{rank}(\mathbf{X})$ represent the trace and the rank of the matrix $\mathbf{X} ; \mathcal{R}(\mathbf{X})$ and $\mathcal{N}(\mathbf{X})$ denote the range and the null space of $\mathbf{X}$; for any $\mathbf{a} \in \mathbb{C}^{m},\|\mathbf{a}\|_{2}^{2} \triangleq \sqrt{\mathbf{a}^{H} \mathbf{a}}$ denotes the Euclidean norm; $\mathbf{A}=\operatorname{diag}\left(\mathbf{A}_{11} \mathbf{A}_{22} \ldots \mathbf{A}_{n n}\right)$ is a diagonal matrix with elements $\mathbf{A}_{i i}$ on the main diagonal; E[.] and $\operatorname{Var}[\cdot]$ denote the statistical mean and variance, respectively; $\operatorname{Re}[\cdot]$ denotes the real part.
}

sensing technique, i.e., $u_{i}(q)=\frac{1}{N} \sum_{k=q N_{s}}^{q N_{s}+N-1}\left|x_{i}(k)\right|^{2}$ [13], where $N=\left\lfloor\tau f_{s}\right\rfloor$ is the number of samples available in the sensing time $\tau, f_{s}$ is the sampling frequency and $N_{s}>N$ denotes the period of the sensing process. According to the Central Limit Theorem, for large $N, u_{i}(q)$ is approximated by a Gaussian random variable (r.v.) under both the hypotheses $\mathcal{H}_{0}$ and $\mathcal{H}_{1}$ [4], [13], [14], with mean $E\left[u_{i}(q) \mid \mathcal{H}_{j}\right] \triangleq \nu_{i \mid \mathcal{H}_{j}}$ and variance $\operatorname{Var}\left[u_{i}(q) \mid \mathcal{H}_{j}\right] \triangleq \zeta_{i \mid \mathcal{H}_{j}}$ given by, respectively [4]:

$\nu_{i \mid \mathcal{H}_{j}}=\left\{\begin{array}{ll}\sigma_{i}^{2} & \mathcal{H}_{0} \\ \left|g_{i}\right|^{2} \breve{\mathcal{E}}_{s}+\sigma_{i}^{2} & \mathcal{H}_{1}\end{array}, \zeta_{i \mid \mathcal{H}_{j}}= \begin{cases}\frac{\sigma_{i}^{4}}{N} & \mathcal{H}_{0} \\ \frac{2\left|g_{i}\right|^{2} \breve{\mathcal{E}}_{s} \sigma_{i}^{2}+\sigma_{i}^{4}}{N} & \mathcal{H}_{1}\end{cases}\right.$

where, $\breve{\mathcal{E}}_{s}=E_{s} / N=\sum_{k=0}^{N-1}|s(k)|^{2} / N$ is the PU transmitted energy normalized with respect to the sensing samples ${ }^{6} N$.

\section{B. The SCD Approach Model}

Let us consider an arbitrary $\mathrm{SU}$, say $\mathrm{SU}_{0}$, acting as decision maker according to the SCD approach and combining $M$ sensing statistics. From Definition 2, it follows that $(M-1)$ sensing statistics are received from $(M-1)$ cooperative SUs, whereas one sensing statistic is locally available. Without loss of generality, let us denote the $(M-1)$ cooperative SUs as $\mathrm{SU}_{2}, \ldots, \mathrm{SU}_{M}$. The base-band signal at $\mathrm{SU}_{0} \mathrm{RF}$ front-end, received from the $i$-th $\mathrm{SU}$, can be written as:

$y_{i}(l)= \begin{cases}u_{0}(l), & \text { if } i=0 \\ \sum_{n=0}^{L_{g_{i}}-1} u_{i}(l-n) h_{i}(n)+n_{i}(l), & \text { if } i \in\{2, \ldots, M\}\end{cases}$

where $l$-th is the arbitrary sampling instant at the $\mathrm{SU}_{0}$ side, $n_{i}(l) \sim \mathcal{C N}\left(0, \delta_{i}^{2}\right)$ is the complex, circular, zero-mean, spatially un-correlated, AWGN at the $\mathrm{SU}_{0}$ side, $\left\{h_{i}(n)\right\}_{n=0}^{L_{g_{i}}-1}$ is the finite multipath channel impulse response with length $L_{g_{i}}$ that models the RC between the $i$-th $\mathrm{SU}$ and $\mathrm{SU}_{0}$, and $u_{0}(l)$ is the $\mathrm{SU}_{0}$ local sensing statistic. It is reasonable to assume that geographically distributed SUs experience independent fading. Therefore, $u_{i}\left(l_{1}\right)$ and $u_{j}\left(l_{2}\right), i \neq j$, are spatially uncorrelated under each hypothesis [4] and independent of the noise too. To simplify the notation, $L_{g}$ denotes the largest channel length, i.e., $L_{g}=\max _{i=2}^{M} L_{g_{i}}$. At $\mathrm{SU}_{0}$, due to the dispersive RCs, we jointly elaborate $L_{e}$ received samples for each $\mathrm{SU}$, i.e., $\mathbf{y}_{i}(l) \triangleq\left[y_{i}(l) y_{i}(l-1) \ldots y_{i}\left(l-L_{e}+1\right)\right]^{T} \in \mathbb{C}^{L_{e}}$, which, by using (3), is equal to:

$$
\mathbf{y}_{i}(l)= \begin{cases}\mathbf{H}_{0} \mathbf{u}_{0}(l), & \text { if } i=0 \\ \mathbf{H}_{i} \mathbf{u}_{i}(l)+\mathbf{n}_{i}(l), & \text { if } i \in\{2, \ldots, M\}\end{cases}
$$

In (4), for $i \neq 0, \mathbf{H}_{i} \in \mathcal{C}^{L_{e} \times\left(L_{e}+L_{g}-1\right)}$ is a Toeplitz upper triangular matrix with first row $\left[h_{i}(0) \ldots h_{i}\left(L_{g}-1\right) 0 \ldots 0\right]$ and first column $\left[h_{i}(0) 0 \ldots 0\right]^{T}, \mathbf{H}_{0} \in \mathcal{R}^{L_{e} \times\left(L_{e}+L_{g}-1\right)}$ is the Toeplitz matrix with first row and first column equal

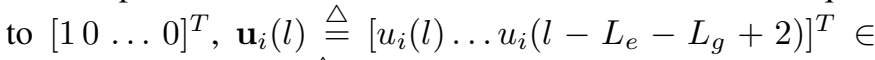
$\mathbb{R}^{L_{e}+L_{g}-1}$ and $\mathbf{n}_{i}(l) \triangleq\left[n_{i}(l) \ldots n_{i}\left(l-L_{e}+1\right)\right]^{T} \in \mathbb{C}^{L_{e}}$. By defining $\mathbf{H}_{\text {sc }} \triangleq \operatorname{diag}\left[\mathbf{H}_{0} \overline{\mathbf{H}}\right]$, with $\overline{\mathbf{H}} \triangleq \operatorname{diag}\left[\mathbf{H}_{2} \ldots \mathbf{H}_{M}\right] \in$

\footnotetext{
${ }^{6} s(k)$ is assumed deterministic and unknown as in [4], [10], [14]. However, if $s(k)$ is modeled as a sequence of i.i.d. r.vv., $u_{i}(q)$ is still approximated by a Gaussian r.v. [13], and the subsequent analysis continues to hold.
} 
$\mathbb{C}^{(M-1) L_{e} \times(M-1)\left(L_{e}+L_{g}-1\right)}, \mathbf{u}_{\mathrm{sc}}(l) \triangleq\left[\mathbf{u}_{0}^{T}(l) \overline{\mathbf{u}}^{T}(l)\right]^{T}$, with $\overline{\mathbf{u}}(l) \triangleq\left[\mathbf{u}_{2}^{T}(l) \ldots \mathbf{u}_{M}^{T}(l)\right]^{T} \in \mathbb{R}^{(M-1)\left(L_{e}+L_{g}-1\right)}, \mathbf{n}_{\mathrm{sc}}(l) \triangleq$ $\left[\mathbf{0}_{L_{e}}^{T} \overline{\mathbf{n}}^{T}(l)\right]^{T}$, with $\overline{\mathbf{n}}(l) \triangleq\left[\mathbf{n}_{2}^{T}(l) \ldots \mathbf{n}_{M}^{T}(l)\right]^{T} \in \mathbb{C}^{(M-1) L_{e}}$, and $\overline{\mathbf{y}}(l) \triangleq\left[\mathbf{y}_{2}^{T}(l) \ldots \mathbf{y}_{M}^{T}(l)\right]^{T},(4)$ is rewritten as ${ }^{7}$ :

$$
\mathbf{y}_{\mathrm{sc}}(l) \in \mathbb{C}^{M L_{e}} \triangleq\left[\mathbf{y}_{0}^{T}(l) \overline{\mathbf{y}}^{T}(l)\right]^{T}=\mathbf{H}_{\mathrm{sc}} \mathbf{u}_{\mathrm{sc}}(l)+\mathbf{n}_{\mathrm{sc}}(l)
$$

By using (2) and (5), the mean $\boldsymbol{\mu}_{\mathrm{sc} \mid \mathcal{H}_{j}} \triangleq E\left[\mathbf{y}_{\mathrm{sc}}(l) \mid \mathcal{H}_{j}\right] \in$ $\mathbb{C}^{M L_{e}}$ and the covariance matrix $\mathbf{C}_{\mathbf{y}_{\mathrm{sc}}} \mid \mathcal{H}_{j} \triangleq E\left[\left(\mathbf{y}_{\mathrm{sc}}(l)-\right.\right.$ $\left.\left.\boldsymbol{\mu}_{\mathrm{sc}}\right)\left(\mathbf{y}_{\mathrm{sc}}(l)-\boldsymbol{\mu}_{\mathrm{sc}}\right)^{H} \mid \mathcal{H}_{j}\right] \in \mathbb{C}^{M L_{e} \times M L_{e}}$ of the Gaussian random vector $\mathbf{y}_{\mathrm{sc}}(l)$ are equal to, respectively:

$$
\begin{gathered}
\boldsymbol{\mu}_{\mathrm{sc} \mid \mathcal{H}_{j}}=\mathbf{H}_{\mathrm{sc}} E\left[\mathbf{u}_{\mathrm{sc}}(l) \mid \mathcal{H}_{j}\right]=\mathbf{H}_{\mathrm{sc}} \boldsymbol{\eta}_{\mathrm{sc}, \mathcal{H}_{j}} \\
\mathbf{C}_{\mathbf{y}_{\mathrm{sc}} \mid \mathcal{H}_{j}}=\mathbf{H}_{\mathrm{sc}} \mathbf{C}_{\mathbf{u}_{\mathrm{sc}} \mid \mathcal{H}_{j}} \mathbf{H}_{\mathrm{sc}}^{H}+\mathbf{R}_{\mathbf{n}_{\mathrm{sc}}}
\end{gathered}
$$

In (6), $\boldsymbol{\eta}_{\text {sc } \mid \mathcal{H}_{j}} \triangleq E\left[\mathbf{u}(l) \mid \mathcal{H}_{j}\right]=\left[\boldsymbol{\eta}_{0 \mid \mathcal{H}_{j}}^{T} \overline{\boldsymbol{\eta}}_{\mathcal{H}_{j}}^{T}\right]^{T}$, with $\overline{\boldsymbol{\eta}}_{\mathcal{H}_{j}}$ defined as $\overline{\boldsymbol{\eta}}_{\mathcal{H}_{j}} \triangleq\left[\boldsymbol{\eta}_{2 \mid \mathcal{H}_{j}}^{T} \ldots \boldsymbol{\eta}_{M \mid \mathcal{H}_{j}}^{T}\right]^{T}$ and $\boldsymbol{\eta}_{k \mid \mathcal{H}_{j}}=$ $\nu_{k \mid \mathcal{H}_{j}} \mathbf{1}_{\left\{L_{e}+L_{g}-1\right\}}$. In (7), $\mathbf{R}_{\mathbf{n}_{\mathrm{sc}}} \triangleq E\left[\mathbf{n}_{\mathrm{sc}}(l) \mathbf{n}_{\mathrm{sc}}^{H}(l)\right] \quad \in$ $\mathbb{R}^{M L_{e} \times M L_{e}}$ and $\mathbf{C}_{\mathbf{u}_{\mathrm{sc}} \mid \mathcal{H}_{j}} \triangleq E\left[\left(\mathbf{u}_{\mathrm{sc}}(l)-\boldsymbol{\eta}_{\mathrm{sc}}\right)\left(\mathbf{u}_{\mathrm{sc}}(l)-\right.\right.$ $\left.\left.\boldsymbol{\eta}_{\text {sc }}\right)^{H} \mid \mathcal{H}_{j}\right] \in \mathbb{R}^{M\left(L_{e}+L_{g}-1\right) \times M\left(L_{e}+L_{g}-1\right)}$ are the noise and the sensing covariance matrices equal to:

$$
\mathbf{R}_{\mathbf{n}_{\mathrm{sc}}}=\operatorname{diag}\left[\mathbf{0}_{L_{e} \times L_{e}} \mathbf{R}_{\mathbf{n}_{2}} \ldots \mathbf{R}_{\mathbf{n}_{M}}\right] \triangleq \operatorname{diag}\left[\mathbf{0}_{L_{e} \times L_{e}} \mathbf{R}_{\overline{\mathbf{n}}}\right]
$$

with $\mathbf{R}_{\mathbf{n}_{i}} \triangleq E\left[\mathbf{n}_{i}(l) \mathbf{n}_{i}^{H}(l)\right]=\delta_{i}^{2} \mathbf{I}_{\left\{L_{e} \times L_{e}\right\}}$ and $\mathbf{R}_{\overline{\mathbf{n}}} \in$ $\mathbb{R}^{(M-1) L_{e} \times(M-1) L_{e}} \triangleq \operatorname{diag}\left[\mathbf{R}_{\mathbf{n}_{2}} \ldots \mathbf{R}_{\mathbf{n}_{M}}\right]$.

$$
\begin{aligned}
\mathbf{C}_{\mathbf{u}_{\mathrm{sc}} \mid \mathcal{H}_{j}} & =\operatorname{diag}\left[\mathbf{C}_{\mathbf{u}_{0} \mid \mathcal{H}_{j}} \mathbf{C}_{\mathbf{u}_{2} \mid \mathcal{H}_{j}} \ldots \mathbf{C}_{\mathbf{u}_{M} \mid \mathcal{H}_{j}}\right] \\
& \triangleq \operatorname{diag}\left[\mathbf{C}_{\mathbf{u}_{0} \mid \mathcal{H}_{j}} \mathbf{C}_{\overline{\mathbf{u}} \mid \mathcal{H}_{j}}\right]
\end{aligned}
$$

with $\mathbf{C}_{\mathbf{u}_{i} \mid \mathcal{H}_{j}} \triangleq E\left[\left(\mathbf{u}_{i}(l)-\boldsymbol{\eta}_{i}\right)\left(\mathbf{u}_{i}(l)-\boldsymbol{\eta}_{i}\right)^{H} \mid \mathcal{H}_{j}\right]=$ $\zeta_{i \mid \mathcal{H}_{j}} \mathbf{I}_{\left\{L_{e}+L_{g}-1\right\}}$ and $\mathbf{C}_{\overline{\mathbf{u}} \mid \mathcal{H}_{j}} \triangleq \operatorname{diag}\left[\mathbf{C}_{\mathbf{u}_{2} \mid \mathcal{H}_{j}} \ldots \mathbf{C}_{\mathbf{u}_{M} \mid \mathcal{H}_{j}}\right]$. By exploiting (5), $\mathbf{C}_{\mathbf{y}_{\mathrm{sc}} \mid \mathcal{H}_{j}}$ in (7) is equivalently expressed as:

$$
\mathbf{C}_{\mathbf{y}_{\mathrm{sc}} \mid \mathcal{H}_{j}}=\left[\begin{array}{cc}
\mathbf{C}_{\mathbf{y}_{0} \mid \mathcal{H}_{j}} & \mathbf{0}_{L_{e} \times(M-1) L_{e}} \\
\mathbf{0}_{(M-1) L_{e} \times L_{e}} & \mathbf{C}_{\overline{\mathbf{y}} \mid \mathcal{H}_{j}}
\end{array}\right]
$$

where

$$
\begin{gathered}
\mathbf{C}_{\mathbf{y}_{0} \mid \mathcal{H}_{j}} \in \mathbb{R}^{L_{e} \times L_{e}} \triangleq \mathbf{H}_{0} \mathbf{C}_{\mathbf{u}_{0} \mid \mathcal{H}_{j}} \mathbf{H}_{0}^{H}=\zeta_{0 \mid \mathcal{H}_{j}} \mathbf{I}_{\left\{L_{e}\right\}} \\
\mathbf{C}_{\overline{\mathbf{y}} \mid \mathcal{H}_{j}} \in \mathbb{C}^{(M-1) L_{e} \times(M-1) L_{e}} \triangleq \overline{\mathbf{H}} \mathbf{C}_{\overline{\mathbf{u}} \mid \mathcal{H}_{j}} \overline{\mathbf{H}}^{H}+\mathbf{R}_{\overline{\mathbf{n}}}
\end{gathered}
$$

Finally, since the pseudo-covariance matrix $\mathbf{C}_{\mathbf{y}_{\mathrm{sc}}^{*} \mid \mathcal{H}_{j}} \triangleq$ $E\left[\left(\mathbf{y}_{\mathrm{sc}}(l)-\boldsymbol{\mu}\right)\left(\mathbf{y}_{\mathrm{sc}}(l)-\boldsymbol{\mu}\right)^{T} \mid \mathcal{H}_{j}\right] \in \mathbb{C}^{M L_{e} \times M L_{e}}$ of $\mathbf{y}_{\mathrm{sc}}(l)$ is not null, $\mathbf{y}_{\mathrm{sc}}(l)$ is improper [12], and $\mathbf{C}_{\mathbf{y}_{\mathrm{sc}}^{*} \mid \mathcal{H}_{j}}$ is given by:

$$
\mathbf{C}_{\mathbf{y}_{\mathrm{sc}}^{*} \mid \mathcal{H}_{j}}=\mathbf{H}_{\mathrm{sc}} \mathbf{C}_{\mathbf{u}_{\mathrm{sc}} \mid \mathcal{H}_{j}} \mathbf{H}_{\mathrm{sc}}^{T}=\left[\begin{array}{cc}
\mathbf{C}_{\mathbf{y}_{0} \mid \mathcal{H}_{j}} & \mathbf{0}_{L_{e} \times(M-1) L_{e}} \\
\mathbf{0}_{(M-1) L_{e} \times L_{e}} & \mathbf{C}_{\overline{\mathbf{y}}^{*} \mid \mathcal{H}_{j}}
\end{array}\right]
$$

where, we exploited the circular property of $\mathbf{n}_{\mathrm{sc}}(l)$, i.e., $\mathbf{R}_{\mathbf{n}_{\mathrm{sc}}^{*}}=0, \mathbf{C}_{\mathbf{y}_{0} \mid \mathcal{H}_{j}}$ is given in (11) and, finally,

$$
\mathbf{C}_{\overline{\mathbf{y}}^{*} \mid \mathcal{H}_{j}} \in \mathbb{C}^{(M-1) L_{e} \times(M-1) L_{e}}=\overline{\mathbf{H}} \mathbf{C}_{\overline{\mathbf{u}} \mid \mathcal{H}_{j}} \overline{\mathbf{H}}^{T}
$$

\footnotetext{
${ }^{7}$ The transmissions of different SUs are assumed orthogonal, [4], [10], [14].
}

\section{The CD Approach Model}

Let us consider now $\mathrm{SU}_{0}$ acting as decision maker according to the $\mathrm{CD}$ approach and combining $M$ sensing statistics. From Definition 1, all the $M$ sensing statistics are received from the $M$ cooperative SUs, denoted without loss of generality as $\mathrm{SU}_{1}, \ldots, \mathrm{SU}_{M}$. Hence, the base-band signal at $\mathrm{SU}_{0} \mathrm{RF}$ frontend received from the $i$-th $\mathrm{SU}$ can be written as:

$$
y_{i}(l)=\sum_{n=0}^{L_{g_{i}}-1} u_{i}(l-n) h_{i}(n)+n_{i}(l), i \in\{1,2, \ldots, M\}
$$

Remark 5. (15) differs from (4) for the presence of the $\mathrm{SU}_{1}$ sensing statistic and the absence of the decision maker sensing statistic $u_{0}(l)$. In fact, according to Definition 1 , the decision maker $\mathrm{SU}_{0}$ does not participate in the sensing of the monitored band and another SU, labeled without loss of generality as $\mathrm{SU}_{1}$, is involved in the cooperative sensing process. We underline that the number of cooperators is fixed to $M$ in both the approaches for a fair comparison. Clearly, the definitions of $\overline{\mathbf{H}}, \overline{\mathbf{u}}(l)$ and $\overline{\mathbf{y}}(l)$, given above, are kept unchanged since they involve the cooperative SUs common to both the approaches, i.e., $\mathrm{SU}_{2}, \ldots, \mathrm{SU}_{M}$.

Similarly to Sec. III-A, by denoting with $\mathbf{H}_{1} \in$ $\mathcal{C}^{L_{e} \times\left(L_{e}+L_{g}-1\right)}$ the Toeplitz upper triangular matrix with first row $\left[h_{1}(0) \ldots h_{1}\left(L_{g}-1\right) 0 \ldots 0\right]$ and first column $\left[h_{1}(0) 0 \ldots 0\right]^{T}, \mathbf{H}_{\mathrm{c}} \triangleq \operatorname{diag}\left[\mathbf{H}_{1} \overline{\mathbf{H}}\right], \mathbf{u}_{c}(l) \triangleq\left[\mathbf{u}_{1}^{T}(l) \overline{\mathbf{u}}^{T}(l)\right]^{T}$, $\mathbf{n}_{c}(l) \triangleq\left[\mathbf{n}_{1}^{T}(l) \overline{\mathbf{n}}^{T}(l)\right]^{T},(15)$ is reformulated as follows:

$$
\mathbf{y}_{c}(l) \in \mathbb{C}^{M L_{e}}=\left[\mathbf{y}_{1}^{T}(l) \overline{\mathbf{y}}^{T}(l)\right]^{T}=\mathbf{H}_{c} \mathbf{u}_{c}(l)+\mathbf{n}_{c}(l)
$$

The mean $\boldsymbol{\mu}_{\mathrm{c} \mid \mathcal{H}_{j}} \in \mathbb{C}^{M L_{e}}$ and covariance matrix $\mathbf{C}_{\mathbf{y}_{c} \mid \mathcal{H}_{j}} \triangleq$ $E\left[\left(\mathbf{y}_{c}(l)-\boldsymbol{\mu}_{c}\right)\left(\mathbf{y}_{c}(l)-\boldsymbol{\mu}_{c}\right)^{H} \mid \mathcal{H}_{j}\right] \in \mathbb{C}^{M L_{e} \times M L_{e}}$ of the Gaussian vector $\mathbf{y}_{c}(l)$ are equal to:

$$
\begin{gathered}
\boldsymbol{\mu}_{\mathrm{c} \mid \mathcal{H}_{j}} \triangleq E\left[\mathbf{y}_{c}(l) \mid \mathcal{H}_{j}\right]=\mathbf{H}_{c} E\left[\mathbf{u}_{c}(l) \mid \mathcal{H}_{j}\right]=\mathbf{H}_{c} \boldsymbol{\eta}_{\mathrm{c}, \mathcal{H}_{j}} \\
\mathbf{C}_{\mathbf{y}_{c} \mid \mathcal{H}_{j}}=\mathbf{H}_{c} \mathbf{C}_{\mathbf{u}_{c} \mid \mathcal{H}_{j}} \mathbf{H}_{c}^{H}+\mathbf{R}_{\mathbf{n}_{c}}= \\
=\left[\begin{array}{cc}
\mathbf{C}_{\mathbf{y}_{1} \mid \mathcal{H}_{j}} & \mathbf{0}_{L_{e} \times(M-1) L_{e}} \\
\mathbf{0}_{(M-1) L_{e} \times L_{e}} & \mathbf{C}_{\overline{\mathbf{y}} \mid \mathcal{H}_{j}}
\end{array}\right]
\end{gathered}
$$

In (17), $\boldsymbol{\eta}_{\mathrm{c} \mid \mathcal{H}_{j}} \triangleq E\left[\mathbf{u}_{c}(l) \mid \mathcal{H}_{j}\right]=\left[\boldsymbol{\eta}_{1 \mid \mathcal{H}_{j}}^{T} \overline{\boldsymbol{\eta}}_{\mathcal{H}_{j}}^{T}\right]^{T}$. In (18), $\mathbf{C}_{\overline{\mathbf{y}} \mid \mathcal{H}_{j}}$ is given by (12), $\mathbf{R}_{\mathbf{n}_{c}} \in \mathbb{R}^{M L_{e} \times M L_{e}} \triangleq E\left[\mathbf{n}_{c}(l) \mathbf{n}_{c}^{H}(l)\right]=$ $\operatorname{diag}\left[\mathbf{R}_{\mathbf{n}_{1}} \mathbf{R}_{\overline{\mathbf{n}}}\right]$ and

$$
\mathbf{C}_{\mathbf{y}_{1} \mid \mathcal{H}_{j}} \in \mathbb{C}^{L_{e} \times L_{e}}=\mathbf{H}_{1} \mathbf{C}_{\mathbf{u}_{1} \mid \mathcal{H}_{j}} \mathbf{H}_{1}^{H}+\mathbf{R}_{\mathbf{n}_{1}}
$$

Finally, the no-null pseudo-covariance matrix $\mathbf{C}_{\mathbf{y}_{c}^{*} \mid \mathcal{H}_{j}} \triangleq$ $E\left[\left(\mathbf{y}_{c}(l)-\boldsymbol{\mu}_{c}\right)\left(\mathbf{y}_{c}(l)-\boldsymbol{\mu}_{c}\right)^{T} \mid \mathcal{H}_{j}\right] \in \mathbb{C}^{M L_{e} \times M L_{e}}$ of $\mathbf{y}_{c}(l)$ is: $\mathbf{C}_{\mathbf{y}_{c}^{*} \mid \mathcal{H}_{j}}=\mathbf{H}_{c} \mathbf{C}_{\mathbf{u}_{c} \mid \mathcal{H}_{j}} \mathbf{H}_{c}^{T}=\left[\begin{array}{cc}\mathbf{C}_{\mathbf{y}_{1}^{*} \mid \mathcal{H}_{j}} & \mathbf{0}_{L_{e} \times(M-1) L_{e}} \\ \mathbf{0}_{(M-1) L_{e} \times L_{e}} & \mathbf{C}_{\overline{\mathbf{y}}^{*} \mid \mathcal{H}_{j}}\end{array}\right]$

with $\mathbf{C}_{\overline{\mathbf{y}}^{*} \mid \mathcal{H}_{j}}$ reported in (14) and $\mathbf{C}_{\mathbf{y}_{1}^{*} \mid \mathcal{H}_{j}}=\mathbf{H}_{1} \mathbf{C}_{\mathbf{u}_{1} \mid \mathcal{H}_{j}} \mathbf{H}_{1}^{T}$.

\section{Cooperative Spectrum Sensing Techniques}

Here, after brief descriptions of the cooperative WL, L and LRT techniques, we specialize them for both the CD and SCD approaches in presence of temporal dispersive RCs. 


\section{A. The Widely Linear Cooperative Technique}

A WL cooperative technique consists in processing jointly, according to a certain criterion, the received vector at the radio front-end of an arbitrary SU and its conjugate version [4], [10], regardless of the decision approach. Hence, by defining the augmented received vector as $\mathbf{y}_{\mathrm{a}}(l) \triangleq\left[\mathbf{y}^{T}(l) \mathbf{y}^{H}(l)\right]^{T} \in$ $\mathbb{C}^{2 M L_{e}}$, and the augmented processing vector as $\mathbf{f}^{T} \triangleq$ $\left[\mathbf{t}^{T} \mathbf{q}^{T}\right]$, the WL test statistic can be written as:

$$
d_{\mathrm{wl}}(l)=\mathbf{t}^{H} \mathbf{y}(l)+\mathbf{q}^{H} \mathbf{y}^{*}(l)=\mathbf{f}^{H} \mathbf{y}_{\mathrm{a}}(l)
$$

with $\mathbf{q}=\mathbf{t}^{*}$, since $\mathbf{f}$ exhibits the conjugate symmetric property for real sensing data $\left\{u_{i}(q)\right\}$, [4]. For this, $d_{\mathrm{wl}}(l)$ results real.

Remark 6. In (21), $\mathbf{y}(l)$ is $\mathbf{y}_{\mathrm{sc}}(l)$ given by (5), if the SCD is adopted, otherwise $\mathbf{y}(l)$ is $\mathbf{y}_{c}(l)$ given by (16). In the following, we omit the subscripts "c" and "sc" for describing the WL strategy regardless of the considered decision approach.

Since $\mathbf{y}(l)$ and $\mathbf{y}^{*}(l)$ are Gaussian, $d_{\mathrm{wl}}(l)$ is Gaussian as well, with mean $\mu_{\mathrm{wl} \mid \mathcal{H}_{j}} \triangleq E\left[d_{\mathrm{wl}}(l) \mid \mathcal{H}_{j}\right]$ and variance $\varphi_{\mathrm{wl} \mid \mathcal{H}_{j}}^{2} \triangleq$ $\operatorname{Var}\left[d_{\mathrm{wl}}(l) \mid \mathcal{H}_{j}\right]$ equal to, respectively:

$$
\begin{gathered}
\mu_{\mathrm{w}|| \mathcal{H}_{j}}=\mathbf{f}^{H} E\left[\mathbf{y}_{\mathrm{a}}(l) \mid \mathcal{H}_{j}\right] \triangleq \mathbf{f}^{H} \widetilde{\boldsymbol{\mu}}_{\mathcal{H}_{j}} \\
\varphi_{\mathrm{wl} \mid \mathcal{H}_{j}}^{2}=\mathbf{f}^{H} E\left[\left(\mathbf{y}_{\mathrm{a}}(l)-\widetilde{\boldsymbol{\mu}}\right)\left(\mathbf{y}_{\mathrm{a}}(l)-\widetilde{\boldsymbol{\mu}}\right)^{H} \mid \mathcal{H}_{j}\right] \mathbf{f} \triangleq \mathbf{f}^{H} \mathbf{C}_{\mathbf{y}_{\mathrm{a}} \mid \mathcal{H}_{j}} \mathbf{f}
\end{gathered}
$$

By denoting with $\mathbf{H}_{\mathrm{a}} \triangleq\left[\mathbf{H}^{T} \mathbf{H}^{H}\right]^{T}, \widetilde{\boldsymbol{\mu}}_{\mathcal{H}_{j}} \in \mathbb{C}^{2 M L_{e}}$ in (22) can be equivalently expressed as:

$$
\widetilde{\boldsymbol{\mu}}_{\mathcal{H}_{j}} \triangleq E\left[\mathbf{y}_{\mathrm{a}}(l) \mid \mathcal{H}_{j}\right]=\mathbf{H}_{\mathrm{a}} \boldsymbol{\eta}_{\mathcal{H}_{j}}=\left[\boldsymbol{\mu}_{\mathcal{H}_{j}}^{T} \boldsymbol{\mu}_{\mathcal{H}_{j}}^{H}\right]^{T}
$$

where, $\mathbf{H}, \boldsymbol{\eta}_{\mathcal{H}_{j}}$ and $\boldsymbol{\mu}_{\mathcal{H}_{j}}$ are given by (5) and (6) if the SCD is adopted, otherwise they are given by (16) and (17). By denoting with $\mathbf{R}_{\mathbf{n}_{a}} \triangleq \operatorname{diag}\left[\mathbf{R}_{\mathbf{n}} \mathbf{R}_{\mathbf{n}}\right] \in \mathbb{R}^{2 M L_{e} \times 2 M L_{e}}$, the augmented covariance matrix $\mathcal{C}_{\mathbf{y}_{a} \mid \mathcal{H}_{j}} \in \mathbb{C}^{2 M L_{e} \times 2 M L_{e}}$ in (23) of the received vector $\mathbf{y}_{\mathrm{a}}$ can be equivalently expressed as:

$$
\mathcal{C}_{\mathbf{y}_{\mathrm{a}} \mid \mathcal{H}_{j}}=\mathbf{H}_{\mathrm{a}} \mathbf{C}_{\mathbf{u} \mid \mathcal{H}_{j}} \mathbf{H}_{\mathrm{a}}^{H}+\mathbf{R}_{\mathbf{n}_{\mathrm{a}}}=\left[\begin{array}{cc}
\mathbf{C}_{\mathbf{y} \mid \mathcal{H}_{j}} & \mathbf{C}_{\mathbf{y}^{*} \mid \mathcal{H}_{j}} \\
\mathbf{C}_{\mathbf{y}^{*} \mid \mathcal{H}_{j}}^{*} & \mathbf{C}_{\mathbf{y} \mid \mathcal{H}_{j}}^{*}
\end{array}\right]
$$

with ${ }^{8} \mathbf{C}_{\mathbf{y} \mid \mathcal{H}_{j}}$ and $\mathbf{C}_{\mathbf{y}^{*} \mid \mathcal{H}_{j}}$ given by (10) and (13) for the SCD, otherwise by (18) and (20). Then, the decision on the PU presence is taken by comparing $d_{\mathrm{wl}}(l)$ with a threshold $\gamma_{\mathrm{wl}}$ :

$$
d_{\mathrm{wl}}(l) \gtreqless \mathcal{H}_{1} \gamma_{0} \gamma_{\mathrm{wl}}
$$

where $\gamma_{\mathrm{wl}}$ can be set to obtain a targeted false-alarm probability [4]. Clearly, the performance of the WL technique depends on the selected optimization criterion used to design f. According to [4], we single out $\mathbf{f}$ by maximizing the Deflection Coefficient (DC), i.e., the variance-normalized distance between the expectations of $d_{\mathrm{wl}}(l)$. To avoid undetermined solutions, the DC is maximized under the $\mathrm{f}$ unit-norm con-

\footnotetext{
${ }^{8}$ Since a covariance matrix is positive definite, except in some degenerate cases of no interest, it is reasonable to assume the covariance matrices $\mathbf{C}_{\mathbf{y} \mid \mathcal{H}_{j}}$ and $\mathbf{C}_{\mathbf{y}^{*} \mid \mathcal{H}_{j}}$ invertible, in both the adopted decision approaches.
}

straint:

$$
\begin{aligned}
& \max _{\mathbf{f}}\left\{m_{\mathrm{wl}}^{2}(\mathbf{f}) \triangleq \frac{\left[\mu_{\mathrm{wl} \mid} \mathcal{H}_{1}-\mu_{\mathrm{wl} \mid} \mathcal{H}_{0}\right]^{2}}{\varphi_{\mathrm{wl} \mid \mathcal{H}_{1}}^{2}}\right\}= \\
&=\max _{\mathbf{f}} \frac{\left[\mathbf{f}^{H}\left(\widetilde{\boldsymbol{\mu}}_{\mathcal{H}_{1}}-\widetilde{\boldsymbol{\mu}}_{\mathcal{H}_{0}}\right)\right]^{2}}{\mathbf{f}^{H} \mathbf{C}_{\mathbf{y}_{\mathrm{a}} \mid \mathcal{H}_{1}} \mathbf{f}}, \text { subject to } \mathbf{f}^{H} \mathbf{f}=1
\end{aligned}
$$

1) The WL Technique for the CD Approach: By adopting the $\mathrm{CD}$ approach, the solution of (27) coincides with the solution derived in [4] for a centralized cooperative sensing architecture. In fact, as underlined in Sec. II, by using the $\mathrm{CD}$ approach in a centralized cooperative sensing architecture, Definition 1 agrees with the traditional decision approach. Hence the system model (16) formally coincides with the system model presented in [4]. For the sake of completeness, here we report the mentioned solution:

$$
\mathbf{f}_{\mathrm{wl-c}}=\left[\begin{array}{c}
\mathbf{t}_{\mathrm{wl-c}} \\
\mathbf{t}_{\mathrm{wl-c}}^{*}
\end{array}\right]=\frac{\mathbf{C}_{\mathbf{y}_{\mathrm{c}, \mathrm{a}} \mid \mathcal{H}_{1}}^{-1}\left(\widetilde{\boldsymbol{\mu}}_{\mathrm{c} \mid \mathcal{H}_{1}}-\widetilde{\boldsymbol{\mu}}_{\mathrm{c} \mid \mathcal{H}_{0}}\right)}{\|\left.\mathbf{C}_{\mathbf{y}_{\mathrm{c}, \mathrm{|}} \mid \mathcal{H}_{1}}^{-1}\left(\widetilde{\boldsymbol{\mu}}_{\mathrm{c} \mid \mathcal{H}_{1}}-\widetilde{\boldsymbol{\mu}}_{\mathrm{c} \mid \mathcal{H}_{0}}\right)\right|_{2} ^{2}}
$$

with $\mathbf{C}_{\mathbf{y}_{\mathrm{c}, \mathrm{a}} \mid \mathcal{H}_{1}}$ and $\widetilde{\boldsymbol{\mu}}_{\mathrm{c} \mid \mathcal{H}_{j}}$ given by (25) and (24), respectively (Remark 6). The maximized DC assured by $\mathbf{f}_{\text {wl-c }}$ is equal to:

$$
m_{\mathrm{wl}-\mathrm{c}}^{2}\left(\mathbf{f}_{\mathrm{wl}-\mathrm{c}}\right)=\left(\widetilde{\boldsymbol{\mu}}_{\mathrm{c} \mid \mathcal{H}_{1}}-\widetilde{\boldsymbol{\mu}}_{\mathrm{c} \mid \mathcal{H}_{0}}\right)^{H} \mathbf{C}_{\mathbf{y}_{\mathrm{c}, \mathrm{a}} \mid \mathcal{H}_{1}}^{-1}\left(\widetilde{\boldsymbol{\mu}}_{\mathrm{c} \mid \mathcal{H}_{1}}-\widetilde{\boldsymbol{\mu}}_{\mathrm{c} \mid \mathcal{H}_{0}}\right)
$$

2) The WL Technique for the SCD Approach: By adopting the SCD approach, we can not proceed as in [4] to determine the solution of (27), since $\mathbf{C}_{\mathbf{y}_{\text {sc.a }} \mid} \mid \mathcal{H}_{j} \in \mathbb{C}^{2 M L_{e} \times 2 M L_{e}}$ is rank-deficient, and hence, not invertible (Proposition 1). In Propositions 1 and 2, we show intermediate results, used in Theorem 1 to derive the solution of (27).

Proposition 1. The rank of the augmented covariance matrix $\mathbf{C}_{\mathbf{y}_{s c, a} \mid \mathcal{H}_{j}}$ is $(2 M-1) L_{e}$.

Proof: See Appendix A.

We observe that the rank-deficiency of $\mathbf{C}_{\mathbf{y}_{\text {sca }} \mid \mathcal{H}_{j}}$ is a consequence of the stacked procedure (Appendix $\mathrm{A}$ and footnote 8).

Proposition 2. The optimized WL processing vector $\mathbf{f}_{\mathrm{wl}-\mathrm{sc}}$, maximizing the deflection coefficient when the SCD approach is adopted, is the solution of the following constrained maximization problem:

$$
\begin{aligned}
& \max _{\mathbf{f}_{s c}} m_{\text {wl-sc }}^{2}\left(\mathbf{f}_{s c}\right)=\max _{\mathbf{f}_{s c}} \frac{\left[\mathbf{f}_{s c}^{H}\left(\widetilde{\boldsymbol{\mu}}_{s c \mid \mathcal{H}_{1}}-\widetilde{\boldsymbol{\mu}}_{s c \mid \mathcal{H}_{0}}\right)\right]^{2}}{\mathbf{f}_{s c}^{H} \mathbf{C}_{\mathbf{y}_{s c, a} \mid \mathcal{H}_{1}} \mathbf{f}_{s c}}, \\
& \text { subject to }\left\{\begin{array}{l}
\mathbf{f}_{s c} \in \mathcal{R}\left(\mathbf{C}_{\mathbf{y}_{s c, a} \mid \mathcal{H}_{1}}\right) \\
\mathbf{f}_{s c}^{H} \mathbf{f}_{s c}=1
\end{array}\right.
\end{aligned}
$$

Proof: See Appendix B

Theorem 1. The optimized WL processing vector $\mathbf{f}_{\mathrm{wl}-\mathrm{sc}}$, maximizing the deflection coefficient when the SCD approach is adopted, has the following expression:

$$
\mathbf{f}_{\mathrm{wl}-\mathrm{sc}}=\left[\begin{array}{c}
\mathbf{t}_{\mathrm{wl}-\mathrm{sc}} \\
\mathbf{t}_{\mathrm{wl}-\mathrm{sc}}^{*}
\end{array}\right]=\frac{\mathbf{C}_{\mathbf{y}_{s c, a} \mid \mathcal{H}_{1}}^{\dagger}\left(\widetilde{\boldsymbol{\mu}}_{s c \mid \mathcal{H}_{1}}-\widetilde{\boldsymbol{\mu}}_{s c \mid \mathcal{H}_{0}}\right)}{\left\|\mathbf{C}_{\mathbf{y}_{s c, a} \mid \mathcal{H}_{1}}\left(\widetilde{\boldsymbol{\mu}}_{s c \mid \mathcal{H}_{1}}-\widetilde{\boldsymbol{\mu}}_{s c \mid \mathcal{H}_{0}}\right)\right\|_{2}^{2}}
$$

where $\mathbf{C}_{\mathbf{y}_{s c, a} \mid \mathcal{H}_{1}}^{\dagger}$ is the Moore-Penrose inverse [11] of the augmented covariance matrix (25) and $\widetilde{\boldsymbol{\mu}}_{s c \mid \mathcal{H}_{j}}$ is given by (24).

Proof: See Appendix C 
By substituting (31) in the DC expression, it results that the maximized DC is equal to:

$$
m_{\mathrm{wl}-\mathrm{sc}}^{2}\left(\mathbf{f}_{\mathrm{wl}-\mathrm{sc}}\right)=\left(\widetilde{\boldsymbol{\mu}}_{\mathrm{sc} \mid \mathcal{H}_{1}}-\widetilde{\boldsymbol{\mu}}_{\mathrm{sc} \mid \mathcal{H}_{0}}\right)^{H} \mathbf{C}_{\mathbf{y}_{\mathrm{sc}, a} \mid \mathcal{H}_{1}}^{\dagger}\left(\widetilde{\boldsymbol{\mu}}_{\mathrm{sc} \mid \mathcal{H}_{1}}-\widetilde{\boldsymbol{\mu}}_{\mathrm{sc} \mid \mathcal{H}_{0}}\right)
$$

\section{B. The Linear Cooperative Technique}

A L cooperative technique consists in processing linearly, according to a certain criterion, the received vector at the radio front-end of an arbitrary SU [4], regardless of the adopted decision approach. By denoting with $\mathbf{w}$ the processing vector and accounting for Remark 6, the L complex test statistic is:

$$
d(l)=\mathbf{w}^{H} \mathbf{y}(l)
$$

The PU decision is taken by comparing the real part of $d(l)$ with a decision threshold $\gamma_{1}[4]$ :

$$
\operatorname{Re}[d(l)] \gtreqless \mathcal{H}_{1} \gamma_{1}
$$

where the real-part operator accounts for the real-nature of the transmitted symbols $\left\{u_{i}(q)\right\}$. Since $\mathbf{y}(l)$ is Gaussian, $\operatorname{Re}[d(l)]$ is Gaussian too. By following similar reasonings as in [4], the mean $\mu_{1 \mid \mathcal{H}_{j}}$ and the variance $\varphi_{1 \mid \mathcal{H}_{j}}^{2}$ of $\operatorname{Re}[d(l)]$ are equal to:

$$
\begin{gathered}
\mu_{1 \mid \mathcal{H}_{j}} \triangleq E\left[\operatorname{Re}[d(l)] \mid \mathcal{H}_{j}\right]=\operatorname{Re}\left(\mathbf{w}^{H} \boldsymbol{\mu}_{\mathcal{H}_{j}}\right) \\
\varphi_{1 \mid \mathcal{H}_{j}}^{2} \triangleq \operatorname{Var}\left[\operatorname{Re}[d(l)] \mid \mathcal{H}_{j}\right]=\frac{\mathbf{w}^{H} \mathbf{C}_{\mathbf{y} \mid \mathcal{H}_{j}} \mathbf{w}+\operatorname{Re}\left[\mathbf{w}^{H} \mathbf{C}_{\mathbf{y}^{*} \mid \mathcal{H}_{j}} \mathbf{w}^{*}\right]}{2}
\end{gathered}
$$

In (35), $\boldsymbol{\mu}_{\mathcal{H}_{j}}$ is given by (6) for the SCD approach, otherwise by (17). In (36), $\mathbf{C}_{\mathbf{y} \mid \mathcal{H}_{j}}$ and $\mathbf{C}_{\mathbf{y}^{*} \mid \mathcal{H}_{j}}$ are given by (10) and (13) for the SCD approach, otherwise by (18) and (20). As for the $\mathrm{WL}$ case, $\mathrm{w}$ is designed to maximize the $\mathrm{DC}$ :

$$
\begin{aligned}
& \max _{\mathbf{w}} m_{1}^{2}(\mathbf{w})=\max _{\mathbf{w}}\left\{\frac{\left|\mathbf{w}^{H}\left(\boldsymbol{\mu}_{\mathcal{H}_{1}}-\boldsymbol{\mu}_{\mathcal{H}_{0}}\right)\right|^{2}}{\mathbf{w}^{H} \mathbf{C}_{\mathbf{y} \mid \mathcal{H}_{1}} \mathbf{w}}\right\} \\
& \text { subject to } \mathbf{w}^{H} \mathbf{w}=1
\end{aligned}
$$

1) The L Technique for the CD Approach: For the reasons underlined for the WL case, by adopting the $\mathrm{CD}$ approach, the solution of (37) coincides with the one derived in [4]. For the sake of completeness, here we report the mentioned solution:

$$
\mathbf{w}_{\mathrm{l}-\mathrm{c}}=\frac{\mathbf{C}_{\mathbf{y}_{c} \mid \mathcal{H}_{1}}^{-1}\left(\boldsymbol{\mu}_{\mathrm{c} \mid \mathcal{H}_{1}}-\boldsymbol{\mu}_{\mathrm{c} \mid \mathcal{H}_{0}}\right)}{\left\|\mathbf{C}_{\mathbf{y}_{c} \mid \mathcal{H}_{1}}^{-1}\left(\boldsymbol{\mu}_{\mathrm{c} \mid \mathcal{H}_{1}}-\boldsymbol{\mu}_{\mathrm{c} \mid \mathcal{H}_{0}}\right)\right\|_{2}^{2}}
$$

where $\boldsymbol{\mu}_{\mathrm{c} \mid \mathcal{H}_{j}}$ and $\mathbf{C}_{\mathbf{y}_{c} \mid \mathcal{H}_{j}}$ are reported in (17) and (18), respectively. The maximized DC assured by $\mathbf{w}_{\mathrm{l}-\mathrm{c}}$ is equal to:

$$
m_{1}^{2}\left(\mathbf{w}_{\mathrm{l}-\mathrm{c}}\right)=\left(\boldsymbol{\mu}_{\mathrm{c} \mid \mathcal{H}_{1}}-\boldsymbol{\mu}_{\mathrm{c} \mid \mathcal{H}_{0}}\right)^{H} \mathbf{C}_{\mathbf{y}_{\mathrm{c}} \mid \mathcal{H}_{1}}^{-1}\left(\boldsymbol{\mu}_{\mathrm{c} \mid \mathcal{H}_{1}}-\boldsymbol{\mu}_{\mathrm{c} \mid \mathcal{H}_{0}}\right)
$$

2) The L Technique for the SCD Approach: By adopting the SCD approach, differently from the WL case, we can proceed similarly to [4] to determine the solution of (37), since $\mathbf{C}_{\mathbf{y}_{\mathrm{sc}}} \mid \mathcal{H}_{j}$ in (7) is invertible (footnote 8). For the sake of brevity we omit every step and we report only the result:

$$
\mathbf{w}_{\mathrm{l}-\mathrm{sc}}=\frac{\mathbf{C}_{\mathbf{y}_{\mathrm{sc}} \mathcal{H}_{1}}^{-1}\left(\boldsymbol{\mu}_{\mathrm{sc} \mid \mathcal{H}_{1}}-\boldsymbol{\mu}_{\mathrm{sc} \mid \mathcal{H}_{0}}\right)}{\left\|\mathbf{C}_{\mathbf{y}_{\mathrm{sc}} \mid \mathcal{H}}^{-1}\left(\boldsymbol{\mu}_{\mathrm{sc} \mid \mathcal{H}_{1}}-\boldsymbol{\mu}_{\mathrm{sc} \mid \mathcal{H}_{0}}\right)\right\|_{2}^{2}}
$$

with $\boldsymbol{\mu}_{\mathrm{sc} \mid \mathcal{H}_{j}}$ and $\mathbf{C}_{\mathbf{y}_{\mathrm{sc}} \mid \mathcal{H}_{j}}$ given by (6) and (7), respectively. By substituting (40) in the DC definition, it results that the maximized DC is equal to:

$$
m_{\mathrm{l}-\mathrm{sc}}^{2}\left(\mathbf{w}_{\mathrm{l}-\mathrm{sc}}\right)=\left(\boldsymbol{\mu}_{\mathrm{sc} \mid \mathcal{H}_{1}}-\boldsymbol{\mu}_{\mathrm{sc} \mid \mathcal{H}_{0}}\right)^{H} \mathbf{C}_{\mathbf{y}_{\mathrm{sc}} \mid \mathcal{H}_{1}}^{-1}\left(\boldsymbol{\mu}_{\mathrm{sc} \mid \mathcal{H}_{1}}-\boldsymbol{\mu}_{\mathrm{sc} \mid \mathcal{H}_{0}}\right)
$$

\section{The LRT-Based Cooperative Technique}

According to the Neyman-Pearson criterion, the optimum test consists in comparing the likelihood ratio of $\mathbf{y}(l)$ with a threshold to make the decision on the PU presence [9].

1) The LRT-Based Technique for the CD Approach: The LRT-based statistic $d_{\mathrm{c}-\mathrm{LRT}}(l)$, after some algebraic manipulations, assumes the quadratic form:

$$
\begin{aligned}
& d_{\mathrm{c}-\mathrm{LRT}}(l)=\mathbf{y}_{\mathrm{c}, \mathrm{a}}^{H}(l)\left[\mathbf{C}_{\mathbf{y}_{\mathrm{c}, \mathrm{a}} \mid \mathcal{H}_{0}}^{-1}-\mathbf{C}_{\mathbf{y}_{\mathrm{c}, \mathrm{a}} \mid \mathcal{H}_{1}}^{-1}\right] \mathbf{y}_{\mathrm{c}, \mathrm{a}}(l)+ \\
& +2 \operatorname{Re}\left[\left(\widetilde{\boldsymbol{\mu}}_{\mathrm{c} \mid \mathcal{H}_{1}}^{H} \mathbf{C}_{\mathbf{y}_{\mathrm{c}, \mathrm{a}} \mid \mathcal{H}_{1}}^{-1}-\widetilde{\boldsymbol{\mu}}_{\mathrm{c} \mid \mathcal{H}_{0}}^{H} \mathbf{C}_{\mathbf{y}_{\mathrm{c}, \mathrm{a}} \mid \mathcal{H}_{0}}^{-1}\right) \mathbf{y}_{\mathrm{c}, \mathrm{a}}(l)\right]
\end{aligned}
$$

with $\mathbf{C}_{\mathbf{y}_{\mathrm{c}, \mathrm{a}} \mid \mathcal{H}_{j}}$ and $\widetilde{\boldsymbol{\mu}}_{\mathrm{c} \mid \mathcal{H}_{j}}$ given by (25) and (24). To derive (42), we used the PDF expression [15] of improper Gaussian random vectors and accounted for the definition of $\mathbf{y}_{\mathrm{c}, \mathrm{a}}(l)$.

2) The LRT-Based Technique for the SCD Approach: The LRT-based statistic $d_{\mathrm{sc}-\mathrm{LRT}}(l)$, after some algebraic manipulations, assumes the quadratic form:

$$
\begin{aligned}
& d_{\mathrm{sc}-\mathrm{LRT}}(l)=\mathbf{y}_{\mathrm{sc}, \mathrm{a}}^{H}(l)\left[\mathbf{C}_{\mathbf{y}_{\mathrm{sc}, \mathrm{a}} \mid \mathcal{H}_{0}}^{\dagger}-\mathbf{C}_{\mathbf{y}_{\mathrm{sc}, \mathrm{a}} \mid \mathcal{H}_{1}}^{\dagger}\right] \mathbf{y}_{\mathrm{sc}, \mathrm{a}}(l)+ \\
& +2 \operatorname{Re}\left[\left(\widetilde{\boldsymbol{\mu}}_{\mathrm{sc} \mid \mathcal{H}_{1}}^{H} \mathbf{C}_{\mathbf{y}_{\mathrm{sc}, \mathrm{a}}^{\dagger} \mid \mathcal{H}_{1}}^{\dagger}-\widetilde{\boldsymbol{\mu}}_{\mathrm{sc} \mid \mathcal{H}_{0}}^{H} \mathbf{C}_{\mathbf{y}_{\mathrm{sc}, \mathrm{a}}}^{\dagger} \mid \mathcal{H}_{0}\right) \mathbf{y}_{\mathrm{sc}, \mathrm{a}}(l)\right]
\end{aligned}
$$

In (43), since $\mathbf{C}_{\mathbf{y}_{\mathrm{sca}, \mathrm{a}} \mid \mathcal{H}_{j}}$ is rank-deficient, we used the degenerate PDF expression [16] of improper Gaussian random vectors.

Remark 7. Since, as well known, finding the optimal threshold for the LRT test is not mathematically tractable, to prove that the LRT-based technique exhibits the threshold behavior as a function of the adopted decision approach we proceed numerically in Sec. VI.

\section{The Decision Approach Threshold Behavior}

Here, we prove the main result of this paper: the detection accuracy exhibits a threshold behavior as a function of the adopted decision approach, regardless of the underlying cooperative sensing architecture. Then, the analytical results are discussed at the end of the section.

\section{A. Threshold Behavior for the WL Cooperative Technique}

To prove the main result of Sec. V-A, i.e., Theorem 2, Proposition 3 is needed. For this, let us introduce the following definitions of the matrices $\bar{\Omega} \in \mathbb{C}^{(M-1) L_{e} \times(M-1) L_{e}}$ and $\boldsymbol{\Omega}_{\mathrm{c}} \in \mathbb{C}^{L_{e} \times L_{e}}$ :

$$
\begin{aligned}
& \overline{\boldsymbol{\Omega}} \triangleq\left(\mathbf{C}_{\overline{\mathbf{y}} \mid \mathcal{H}_{1}}-\mathbf{C}_{\overline{\mathbf{y}}^{*} \mid \mathcal{H}_{1}}\left(\mathbf{C}_{\overline{\mathbf{y}} \mid \mathcal{H}_{1}}^{*}\right)^{-1} \mathbf{C}_{\overline{\mathbf{y}}^{*} \mid \mathcal{H}_{1}}^{*}\right)^{-1} \\
& \boldsymbol{\Omega}_{\mathrm{c}} \triangleq\left(\mathbf{C}_{\mathbf{y}_{1} \mid \mathcal{H}_{1}}-\mathbf{C}_{\mathbf{y}_{1}^{*} \mid \mathcal{H}_{1}}\left(\mathbf{C}_{\mathbf{y}_{1} \mid \mathcal{H}_{1}}^{*}\right)^{-1} \mathbf{C}_{\mathbf{y}_{1}^{*} \mid \mathcal{H}_{1}}^{*}\right)^{-1}
\end{aligned}
$$

with $\mathbf{C}_{\overline{\mathbf{y}} \mid \mathcal{H}_{j}}, \mathbf{C}_{\overline{\mathbf{y}}^{*} \mid \mathcal{H}_{j}}, \mathbf{C}_{\mathbf{y}_{1} \mid \mathcal{H}_{j}}$ and $\mathbf{C}_{\mathbf{y}_{1}^{*} \mid \mathcal{H}_{j}}$ given by (12), (14), (19) and (20), respectively.

Proposition 3. The optimized WL sub-vectors $\mathbf{t}_{\mathrm{wl-c}}$ and $\mathbf{t}_{\mathrm{wl}-\mathrm{sc}}$ for the CD and SCD approaches, given in (28) and (31), 
respectively, can be expressed as:

$$
\begin{aligned}
& \mathbf{t}_{\mathrm{wl-c}}=\frac{\boldsymbol{\Sigma}_{\mathrm{wl-c}}\left(\boldsymbol{\eta}_{c \mid \mathcal{H}_{1}}-\boldsymbol{\eta}_{c \mid \mathcal{H}_{0}}\right)}{\sqrt{2} \|\left.\boldsymbol{\Sigma}_{\mathrm{wl-c}}\left(\boldsymbol{\eta}_{c \mid \mathcal{H}_{1}}-\boldsymbol{\eta}_{c \mid \mathcal{H}_{0}}\right)\right|_{2} ^{2}} \\
& \mathbf{t}_{\mathrm{wl}-\mathrm{sc}}=\frac{\boldsymbol{\Sigma}_{\mathrm{wl-sc}}\left(\boldsymbol{\eta}_{s c \mid \mathcal{H}_{1}}-\boldsymbol{\eta}_{s c \mid \mathcal{H}_{0}}\right)}{\sqrt{2}\left\|\boldsymbol{\Sigma}_{\mathrm{wl}-\mathrm{sc}}\left(\boldsymbol{\eta}_{s c \mid \mathcal{H}_{1}}-\boldsymbol{\eta}_{s c \mid \mathcal{H}_{0}}\right)\right\|_{2}^{2}}
\end{aligned}
$$

with $\boldsymbol{\eta}_{c \mid \mathcal{H}_{j}}$ and $\boldsymbol{\eta}_{s c \mid \mathcal{H}_{j}}$ reported in (17) and (6), respectively, and $\boldsymbol{\Sigma}_{\mathrm{wl-c}}$ and $\boldsymbol{\Sigma}_{\mathrm{wl}-\mathrm{sc}}$ equal to (46) and (47), respectively, reported at the top of the next page. In (46) and (47), $\mathbf{C}_{\mathbf{y}_{0} \mid \mathcal{H}_{j}}$ is given in (12), $\mathbf{\mathbf { H }}$ and $\mathbf{H}_{1}$ are defined in Sec. III.

Proof: See Appendix D.

Theorem 2. The WL cooperative technique implemented according to the SCD approach assures higher (lower) detection accuracy than the WL cooperative technique implemented according to the $C D$ approach if and only if $\theta_{\mathrm{wl}}>1\left(\theta_{\mathrm{wl}}<1\right)$,

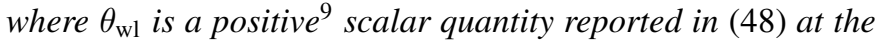
top of the next page. In (48), $\nu_{k \mid \mathcal{H}_{j}}$ and $\zeta_{k \mid \mathcal{H}_{j}}$ are given by (2).

\section{Proof: See Appendix E.}

Remark 8. As proved in Appendix E, when $\theta_{\mathrm{wl}}=1$ the detection accuracy assured by the WL cooperative technique implemented according to the $\mathrm{CD}$ approach coincides with the detection accuracy assured by the WL technique implemented according to the SCD approach. Thus, $\theta_{\mathrm{wl}}=1$ constitutes the searched threshold value of the WL detection accuracy.

Corollary 1. The threshold $\theta_{\mathrm{wl}}$ (48) depends only on the statistics and the channel characteristics of $S U_{0}$ and $S U_{1}$.

Proof: It follows directly from the proof of Theorem 2 given in Appendix E.

Remark 9. The results of Corollary 1 agree with the intuition. In fact, the $(M-1)$ cooperative SUs, denoted with $\mathrm{SU}_{2}, \ldots, \mathrm{SU}_{M}$, affect the performance of both the $\mathrm{CD}$ and SCD implementations of the WL technique in the same way. This means that, although the performance of both the implementations improves as the number $M$ of cooperative SUs grows, the threshold value does not change, as confirmed by the numerical results in Sec. VI.

Remark 10. From (48), it is immediate to verify that if $L_{g_{1}} \rightarrow \infty$ (with $L_{e}<\infty$ ), i.e., if the RC established in the $\mathrm{CD}$ approach between $\mathrm{SU}_{1}$ and $\mathrm{SU}_{0}$ has a infinite memory, $\theta_{\mathrm{wl}} \rightarrow 0$. This means that the $\mathrm{CD}$ approach outperforms the SCD one, i.e., it assures higher detection accuracy than the SCD approach, regardless of the $\mathrm{SU}_{1}$ sensing channel conditions. In other words, it is always more advantageous in terms of detection accuracy involving another SU in the cooperative process rather than using the local sensing statistic of the decision maker $\mathrm{SU}_{0}$, independently of the sensing channel conditions. This surprising result suggests that frequencyselective fading on the RC introduces degrees of freedom that the $\mathrm{CD}$ approach is able to exploit. In practice, it is enough $L_{g_{1}}>>1$, in the order of 10 in operative conditions of practical interest, to assure this behavior (see Sec. VI).

Remark 11. From (48), it results that if the noise power on the $\mathrm{SU}_{0}$ sensing channel goes to infinity, $\sigma_{0}^{2} \rightarrow \infty, \theta_{\mathrm{wl}} \rightarrow 0$,

\footnotetext{
${ }^{9}$ The positive nature of $\theta_{\mathrm{wl}}$ is discussed in Appendix E.
}

i.e., the $\mathrm{CD}$ approach assures higher detection accuracy than the SCD one, regardless of the $\mathrm{SU}_{1} \mathrm{RC}$ conditions. Hence, it is more convenient involving $\mathrm{SU}_{1}$ in the cooperative process rather than using the local statistic of $\mathrm{SU}_{0}$, affected by deep noise. Clearly, if $\sigma_{1}^{2} \rightarrow \infty, \theta_{\mathrm{wl}} \rightarrow \infty$, i.e., the CD approach behavior is inverted with respect to the one described. In practice, it is enough $\sigma_{1}^{2}>>\sigma_{0}^{2}$, to assure this behavior in operative conditions of practical interest (see Sec. VI ).

Remark 12. When the noise power $\delta_{1}^{2}$ of $\mathrm{SU}_{1}$ at $\mathrm{SU}_{0}$ side goes to infinite, i.e., $\delta_{1}^{2} \rightarrow+\infty, \theta_{\mathrm{wl}} \rightarrow+\infty$ as well, i.e., the SCD approach outperforms the CD one. This suggests that if the RC noise power increases significantly, it is more advantageous using the sensing statistic of $\mathrm{SU}_{0}$, not affected by the RC impairments, than involving another $\mathrm{SU}$ in the cooperative process, independently of the SC conditions. In practice, it is enough $\delta_{1}^{2}>>0$ to assure this behavior in operative conditions of practical interest (see Sec. VI).

Corollary 2. If $L_{g_{1}} \rightarrow 1$, i.e., if the $R C$ established between $S U_{1}$ and $S U_{0}$ in the $C D$ approach is not temporal dispersive, we have:

$$
\lim _{L_{g_{1}} \rightarrow 1} \theta_{\mathrm{wl}}=\frac{\left|g_{0}\right|^{4} \zeta_{0 \mid \mathcal{H}_{1}}^{-1}}{\left|g_{1}\right|^{4} \frac{2\left|h_{1}\right|^{2}}{2\left|h_{1}\right|^{2} \zeta_{1 \mid \mathcal{H}_{1}}+\delta_{1}^{2}}}
$$

Proof: By using (19), (20) and $\mathbf{H}_{1}=h_{1} \mathbf{I}_{L_{e}}$ when $L_{g_{1}} \rightarrow$ 1, (49) is achieved.

Remark 13. From (49) it results that:

$$
\lim _{\delta_{1}^{2} \rightarrow 0}\left\{\lim _{L_{g_{1}} \rightarrow 1} \theta_{\mathrm{wl}}\right\}=\frac{\left|g_{0}\right|^{4} \zeta_{0 \mid \mathcal{H}_{1}}^{-1}}{\left|g_{1}\right|^{4} \zeta_{1 \mid \mathcal{H}_{1}}^{-1}}
$$

Therefore, when the $\mathrm{RC}$ between $\mathrm{SU}_{1}$ and $\mathrm{SU}_{0}$ is ideal, the detection accuracy threshold depends only on the sensing channel propagation effects of $\mathrm{SU}_{0}$ and $\mathrm{SU}_{1}$, as expected.

\section{B. Threshold Behavior for the L Cooperative Technique}

To prove the main result of Sec. V-B, i.e., Theorem 3, Proposition 4 is needed.

Proposition 4. The optimized L vectors $\mathrm{w}_{\mathrm{l}-\mathrm{c}}$ and $\mathrm{w}_{\mathrm{l}-\mathrm{sc}}$ for the $C D$ and the SCD approaches, given in (38) and (40), respectively, can be expressed as:

$$
\begin{array}{r}
\mathbf{w}_{1-\mathrm{c}}=\frac{\boldsymbol{\Sigma}_{\mathrm{l-c}}\left(\boldsymbol{\eta}_{c \mid \mathcal{H}_{1}}-\boldsymbol{\eta}_{c \mid \mathcal{H}_{0}}\right)}{\left\|\boldsymbol{\Sigma}_{\mathrm{l}-\mathrm{c}}\left(\boldsymbol{\eta}_{c \mid \mathcal{H}_{1}}-\boldsymbol{\eta}_{c \mid \mathcal{H}_{0}}\right)\right\|_{2}^{2}} \\
\mathbf{w}_{\mathrm{l}-\mathrm{sc}}=\frac{\boldsymbol{\Sigma}_{\mathrm{l}-\mathrm{sc}}\left(\boldsymbol{\eta}_{s c \mid \mathcal{H}_{1}}-\boldsymbol{\eta}_{s c \mid \mathcal{H}_{0}}\right)}{\left\|\boldsymbol{\Sigma}_{\mathrm{l}-\mathrm{sc}}\left(\boldsymbol{\eta}_{s c \mid \mathcal{H}_{1}}-\boldsymbol{\eta}_{s c \mid \mathcal{H}_{0}}\right)\right\|_{2}^{2}}
\end{array}
$$

with $\boldsymbol{\eta}_{c \mid \mathcal{H}_{j}}$ and $\boldsymbol{\eta}_{s c \mid \mathcal{H}_{j}}$ reported in (17) and (6), respectively, and $\boldsymbol{\Sigma}_{1-\mathrm{c}}$ and $\boldsymbol{\Sigma}_{1-\mathrm{sc}}$ equal to:

$$
\begin{aligned}
\boldsymbol{\Sigma}_{\mathrm{l-c}} & =\left[\begin{array}{cc}
\mathbf{C}_{\mathbf{y}_{1 \mid \mathcal{H}}}^{-1} \mathbf{H}_{1} & \mathbf{0}_{L_{e} \times(M-1)\left(L_{e}+L_{g}-1\right)} \\
\mathbf{0}_{(M-1) L_{e} \times\left(L_{e}+L_{g}-1\right)} & \mathbf{C}_{\overline{\mathbf{y}} \mid \mathcal{H}_{1}}^{-1} \overline{\mathbf{H}}
\end{array}\right] \\
\boldsymbol{\Sigma}_{\mathrm{l}-\mathrm{sc}} & =\left[\begin{array}{cc}
\mathbf{C}_{\mathbf{y}_{0 \mid \mathcal{H}_{1}}}^{-1} \mathbf{H}_{0} & \mathbf{0}_{L_{e} \times(M-1)\left(L_{e}+L_{g}-1\right)} \\
\mathbf{0}_{(M-1) L_{e} \times\left(L_{e}+L_{g}-1\right)} & \mathbf{C}_{\overline{\mathbf{y}} \mid \mathcal{H}_{1}}^{-1} \overline{\mathbf{H}}
\end{array}\right]
\end{aligned}
$$

Proof: The proof follows after some algebraic manipulations, by exploiting (38) and (40) and by using the lemma for the inverse of a partitioned matrix [17]. 


$$
\begin{aligned}
& \boldsymbol{\Sigma}_{\mathbf{w l - c}}=\left[\begin{array}{cc}
\boldsymbol{\Omega}_{\mathbf{c}} \mathbf{H}_{1}-\mathbf{C}_{\mathbf{y}_{1} \mid \mathcal{H}_{1}}^{-1} \mathbf{C}_{\mathbf{y}_{1}^{*} \mid \mathcal{H}_{1}} \boldsymbol{\Omega}_{\mathrm{c}}^{*} \mathbf{H}_{1}^{*} & \mathbf{0}_{L_{e} \times(M-1)\left(L_{e}+L_{g}-1\right)} \\
\mathbf{0}_{(M-1) L_{e} \times\left(L_{e}+L_{g}-1\right)} & \overline{\boldsymbol{\Omega}} \overline{\mathbf{H}}-\mathbf{C}_{\overline{\mathbf{y}} \mid \mathcal{H}_{1}}^{-1} \mathbf{C}_{\overline{\mathbf{y}}^{*} \mid \mathcal{H}_{1}} \overline{\mathbf{\Omega}}^{*} \overline{\mathbf{H}}^{*}
\end{array}\right] \\
& \boldsymbol{\Sigma}_{\text {wl-sc }}=\left[\begin{array}{cc}
\frac{1}{2} \mathbf{C}_{\mathbf{y}_{0} \mid \mathcal{H}_{1}}^{-1} \mathbf{H}_{0} & \mathbf{0}_{L_{e} \times(M-1)\left(L_{e}+L_{g}-1\right)} \\
\mathbf{0}_{(M-1) L_{e} \times\left(L_{e}+L_{g}-1\right)} & \overline{\mathbf{\Omega}} \overline{\mathbf{H}}-\mathbf{C}_{\overline{\mathbf{y}} \mid \mathcal{H}_{1}}^{-1} \mathbf{C}_{\overline{\mathbf{y}}^{*} \mid \mathcal{H}_{1}} \overline{\mathbf{\Omega}}^{*} \overline{\mathbf{H}}^{*}
\end{array}\right] \\
& \theta_{\mathrm{wl}} \triangleq \frac{\left(\nu_{0 \mid \mathcal{H}_{1}}-\nu_{0 \mid \mathcal{H}_{0}}\right)^{2} \zeta_{0 \mid \mathcal{H}_{1}}^{-1} L_{e}}{2\left(\nu_{1 \mid \mathcal{H}_{1}}-\nu_{1 \mid \mathcal{H}_{0}}\right)^{2} \mathbf{1}_{\left\{L_{e}+L_{g}-1\right\}}^{T} \operatorname{Re}\left[\mathbf{H}_{1}^{H}\left(\boldsymbol{\Omega}_{\mathrm{c}} \mathbf{H}_{1}-\mathbf{C}_{\mathbf{y}_{1} \mid \mathcal{H}_{1}}^{-1} \mathbf{C}_{\mathbf{y}_{1}^{*} \mid \mathcal{H}_{1}} \boldsymbol{\Omega}_{\mathrm{c}}^{*} \mathbf{H}_{1}^{*}\right)\right] \mathbf{1}_{\left\{L_{e}+L_{g}-1\right\}}}
\end{aligned}
$$

Theorem 3. The $L$ cooperative technique implemented according to the SCD approach assures higher (lower) detection accuracy than the $L$ cooperative technique implemented according to the CD approach, if and only if $\theta_{1}>1\left(\theta_{1}<1\right)$, where $\theta_{1}$ is a positive scalar quantity equal to:

$\theta_{1} \triangleq \frac{\left(\nu_{0 \mid \mathcal{H}_{1}}-\nu_{0 \mid \mathcal{H}_{0}}\right)^{2} \zeta_{0 \mid \mathcal{H}_{1}}^{-1} L_{e}}{\left(\nu_{1 \mid \mathcal{H}_{1}}-\nu_{1 \mid \mathcal{H}_{0}}\right)^{2} \mathbf{1}_{\left\{L_{e}+L_{g}-1\right\}}^{T} \mathbf{H}_{1}^{H} \mathbf{C}_{\mathbf{y}_{1} \mid \mathcal{H}_{1}}^{-1} \mathbf{H}_{1} \mathbf{1}_{\left\{L_{e}+L_{g}-1\right\}}}$

Proof: The proof is achieved by reasoning as for Theorem 2 and by using (51).

Remark 14. Similarly to the WL case, when $\theta_{1}=1$ the detection accuracy assured by the $\mathrm{L}$ cooperative technique implemented according to the $\mathrm{CD}$ approach coincides with the detection accuracy assured by the $\mathrm{L}$ technique implemented according to the SCD approach. Thus, $\theta_{1}=1$ constitutes the searched threshold value of the $\mathrm{L}$ detection accuracy.

Corollary 3. The threshold $\theta_{1}$ (54) depends only on the statistics and the channel characteristics of $S U_{0}$ and $S U_{1}$.

Proof: It follows directly from Theorem 3.

Remark 15. The results of Corollary 3 agree with the intuition. In fact, the $(M-1)$ cooperative SUs, denoted with $\mathrm{SU}_{2}, \ldots, \mathrm{SU}_{M}$, affect the performance of both the $\mathrm{CD}$ and SCD implementations of the L technique in the same way. This means that, although the performance of both the implementations improves as the number $M$ of cooperative SUs grows, the threshold value does not change, as confirmed by the numerical results in Sec. VI. Moreover, Remarks 10, 11 and 12 hold also for the $\mathrm{L}$ cooperative technique.

Corollary 4. If $L_{g_{1}} \rightarrow 1$, i.e., if the RC established between $S U_{1}$ and $S U_{0}$ in the $C D$ approach is not temporal dispersive, we have:

$$
\lim _{L_{g_{1}} \rightarrow 1} \theta_{1}=\frac{\left|g_{0}\right|^{4} \zeta_{0 \mid \mathcal{H}_{1}}^{-1}}{\left|g_{1}\right|^{4} \frac{\left|h_{1}\right|^{2}}{\left|h_{1}\right|^{2} \zeta_{1 \mid \mathcal{H}_{1}}+\delta_{1}^{2}}}
$$

Proof: The proof follows the same guidelines used for Corollary 2.

If $\delta_{1}^{2} \rightarrow 0, \theta_{1}$ is given by (50) and Remark 13 still holds.

\section{Result Discussion}

Stemming from the developed analysis, we can make some considerations. For example, if the local noise levels on the SCs and/or the RCs are known a priori (possibly from experimental measurements when the primary system is turned

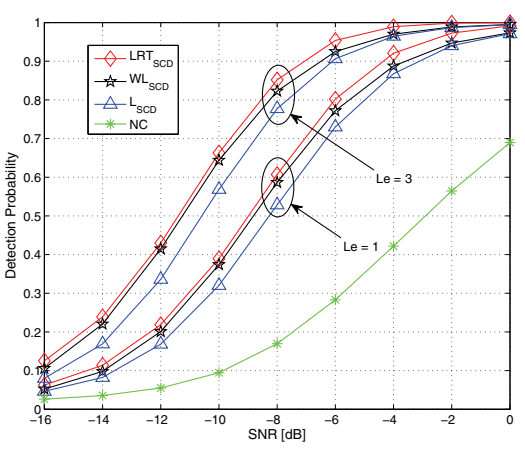

Fig. 2. $\quad P_{\text {det }}$ vs SNR, $M=4, L_{g}=4, N=40$.

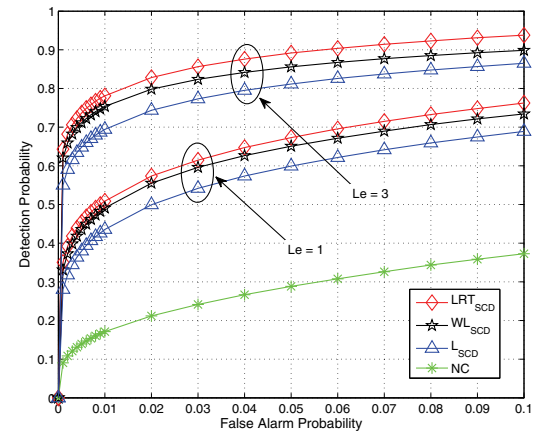

Fig. 3. $P_{d e t}$ vs $P_{f}, M=4, L_{g}=4, N=40$.

off, or from some previous experience), then it is possible to have a-priori insights to choose the decision approach for an arbitrary cooperative technique. In other words, having some a-priori information on the environment provides guidelines on the decision approach to adopt. This is very important, since the choice between the two approaches affects not only the detection accuracy of an arbitrary cooperative sensing technique, but also the entire CR network efficiency/performance, involving crucial trade-off reasonings. In fact, in the $\mathrm{CD}$ approach, the decision maker is not involved in the sensing process, thus being able to perform other CR tasks during its cooperators' sensing time. However, since another SU is involved in the cooperative process, the coordination cost increases. Differently, in the SCD approach, the decision maker is directly involved in the sensing process. Consequently, the coordination cost decreases but the decision maker cannot perform other CR tasks during its cooperators' sensing time. 


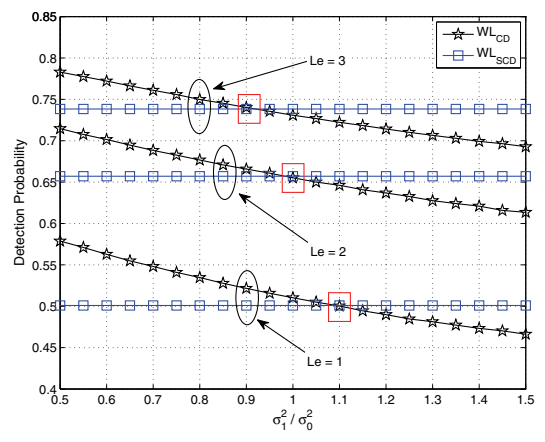

Fig. 4. $\quad P_{\text {det }}$ vs $\frac{\sigma_{1}^{2}}{\sigma_{0}^{2}}$, WL strategy, $P_{f}=10^{-2}$.

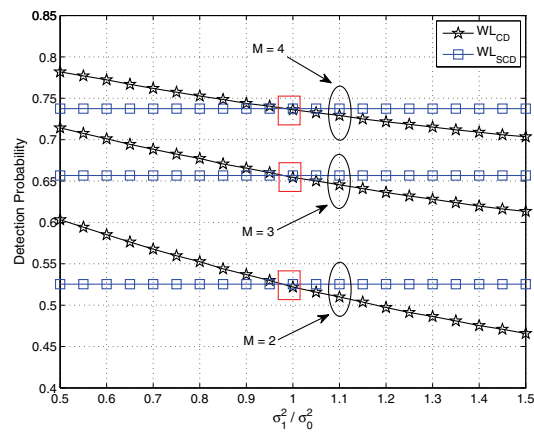

Fig. 7. $P_{\text {det }}$ vs $\sigma_{1}^{2} / \sigma_{0}^{2}$, WL strategy, $P_{f}=10^{-2}$

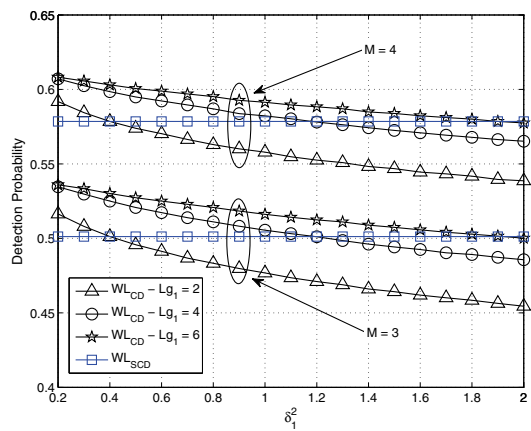

Fig. 10. $P_{\text {det }}$ vs $\delta_{1}^{2}$, WL strategy.

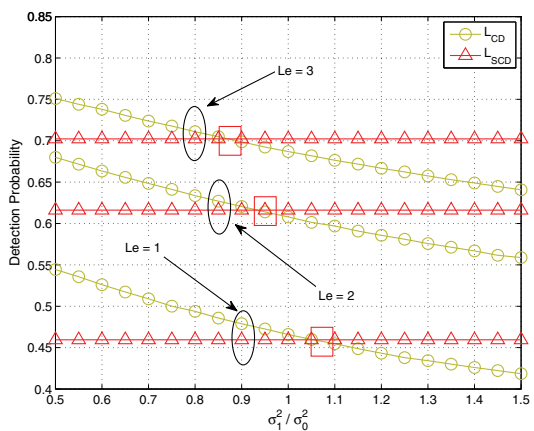

Fig. 5. $\quad P_{\text {det }}$ vs $\frac{\sigma_{1}^{2}}{\sigma_{0}^{2}}, \mathrm{~L}$ strategy, $P_{f}=10^{-2}$.

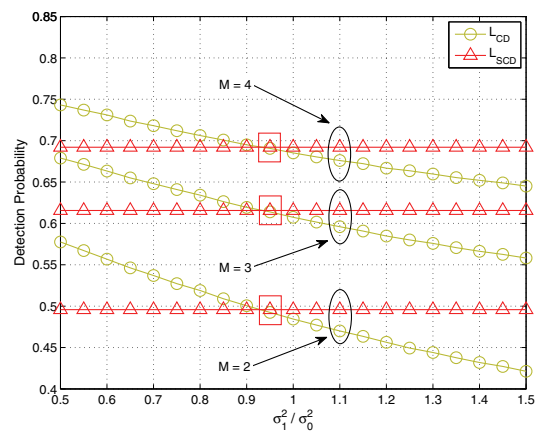

Fig. 8. $P_{\text {det }}$ vs $\sigma_{1}^{2} / \sigma_{0}^{2}$, L strategy, $P_{f}=10^{-2}$

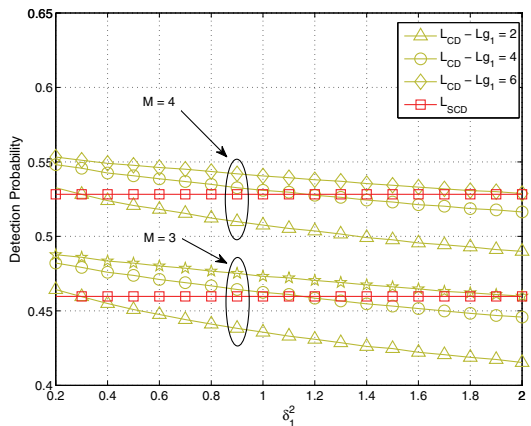

Fig. 11. $P_{\text {det }}$ vs $\delta_{1}^{2}$, L strategy.

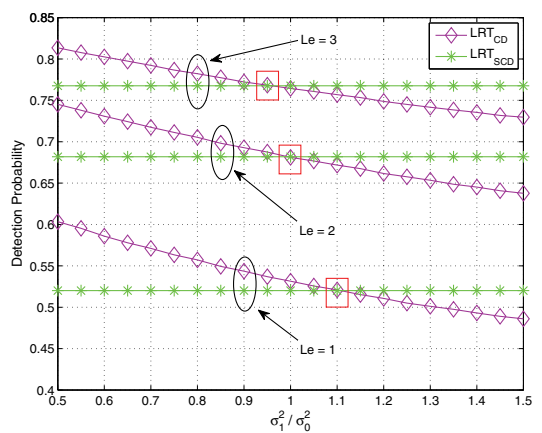

Fig. 6. $P_{\text {det }}$ vs $\frac{\sigma_{1}^{2}}{\sigma_{0}^{2}}$, LRT strategy, $P_{f}=10^{-2}$.

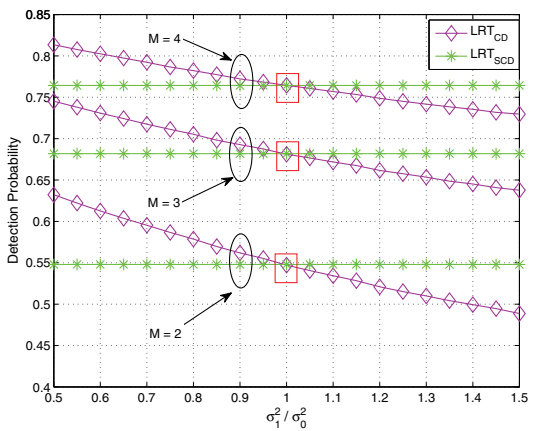

Fig. 9. $P_{\text {det }}$ vs $\sigma_{1}^{2} / \sigma_{0}^{2}$, LRT strategy, $P_{f}=10^{-2}$

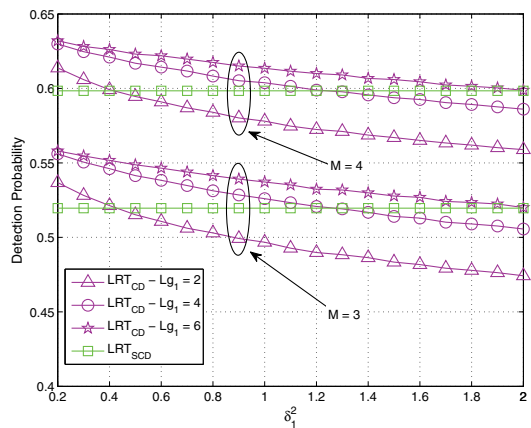

Fig. 12. $P_{\text {det }}$ vs $\delta_{1}^{2}$, LRT strategy.

\section{PERformance EVAluation}

Here, we validate the derived analytical results by extensive Monte Carlo simulations. Specifically, first, we validate the proposed WL and L cooperative sensing techniques implemented by adopting the SCD approach ${ }^{10}$, then we validate the existence of the threshold behavior in the detection accuracy as a function of the adopted decision approach, for all the sensing techniques considered in Sec. IV. Each experiment consists in $10^{6}$ independent Monte Carlo runs, and in each run the noise samples, the sensing channels $\left\{g_{i}\right\}_{i=1}^{M}$ and the tap values of the FIR filters, modeling the RCs, are randomly and independently generated according to a zero-mean complex Gaussian distribution. For simplicity, $s(k)=1$. The average SNR at each

${ }^{10}$ The WL and $\mathrm{L}$ cooperative sensing techniques implemented by adopting the CD approach do not need to be validated, since as highlighted in Sec. IV, they perform as the techniques in [4].
SU is defined as [4], [14]: $\left\{\mathrm{SNR}_{i}\right\}_{i=1}^{M}=E\left[\left|g_{i}\right|^{2}\right] E_{s} /\left(N \sigma_{i}^{2}\right)$.

Experiment 1: Fig. 2 shows the detection probability $P_{\text {det }}$ versus (vs) the SNR, for a fixed value of the false-alarm probability $P_{f}=10^{-2}$ and for two different values of $L_{e}{ }^{11}$. The noise variances of each $\mathrm{SU}$ at $\mathrm{SU}_{0}$ side are set equal to $\delta_{2}^{2}=0.1, \delta_{3}^{2}=0.2$ and $\delta_{4}^{2}=0.4$. Moreover, an additional noncooperative (NC) sensing technique is considered, in which $\mathrm{SU}_{0}$ takes the decision based only on its local energy. The results show that the cooperative techniques perform notably better than the NC one, as expected, and that the WL detector outperforms the $\mathrm{L}$ one in operative conditions of practical

${ }^{11}$ Let us recall the main notations adopted through the paper: $L_{e}$ is the number of jointly elaborated received samples for each $\mathrm{SU}, N$ is the number of samples available in the sensing time, $M$ is the number of cooperative SUs, $L_{g_{i}}$ is the length of the finite multi-path channel impulse response between the $i$-th $\mathrm{SU}$ and $\mathrm{SU}_{0}, L_{g} \triangleq \max _{i=2}^{M} L_{g_{i}}, \delta_{i}^{2}$ is the noise variance for the $i$-th RC, and finally $\sigma_{i}^{2}$ is the noise variance for the $i$-th sensing channel. 

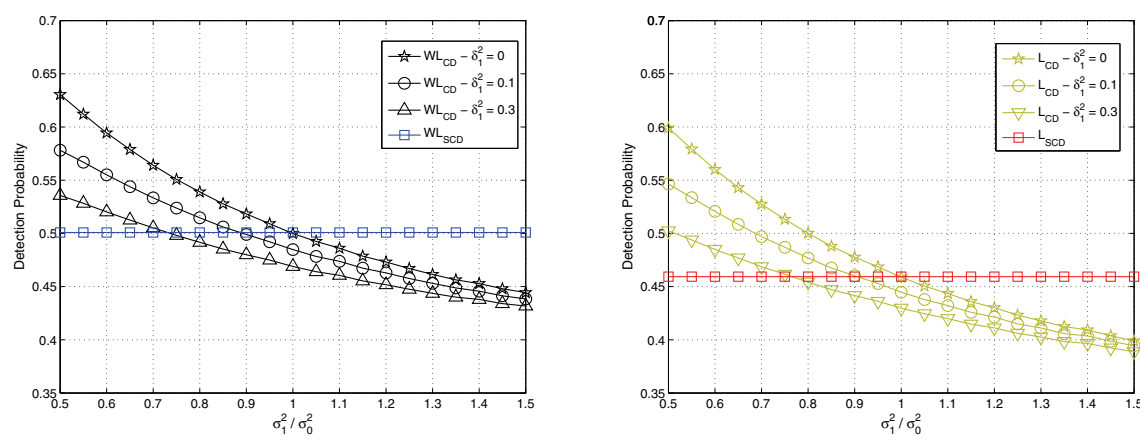

Fig. 13. $\quad P_{\text {det }}$ vs $\sigma_{1}^{2} / \sigma_{0}^{2}$, WL strategy, $L_{g_{1}}=1$. Fig. 14. $\quad P_{\text {det }}$ vs $\sigma_{1}^{2} / \sigma_{0}^{2}$, L strategy, $L_{g_{1}}=1$.

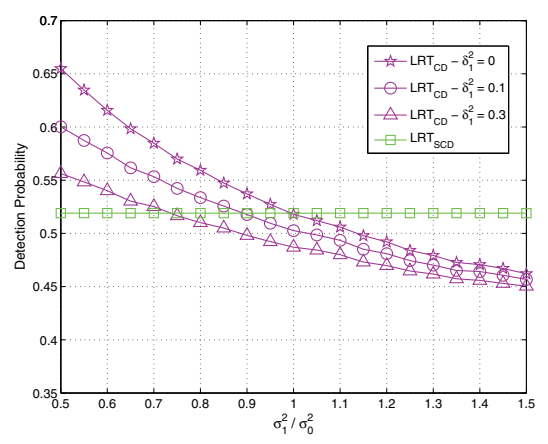

Fig. 15. $\quad P_{\text {det }}$ vs $\sigma_{1}^{2} / \sigma_{0}^{2}$, LRT strategy, $L_{g_{1}}=1$. interest, i.e., for low SNR values, assuring so less interference on the PU. The WL gain is justified by its capacity to exploit the information contained in the statistical pseudocovariance of the received signal. Plus, for both the $L_{e}$ values, the WL detector performs almost comparable with the optimal LRT, but with a significantly lower computational complexity.

Experiment 2: Fig. 3 shows $P_{d e t}$ vs $P_{f}$, with the same simulation setting of Experiment 1. The SNR is different for each SU to analyze how different sensing capabilities can affect the global decision, and it is set as: $\mathrm{SNR}_{0}=-8 \mathrm{~dB}$, $\mathrm{SNR}_{2}=-8.6 \mathrm{~dB}, \mathrm{SNR}_{3}=-9.2 \mathrm{~dB}, \mathrm{SNR}_{4}=-10 \mathrm{~dB}$. The results confirm the performance gain of the $\mathrm{WL}$ detector with respect to the $\mathrm{L}$ one, and the capability of the cooperative strategies, implemented by adopting the SCD approach, to counteract the channel impairments.

In the following, we conduct several experiments to substantiate the threshold behavior of the detection accuracy as a function of the adopted decision approach, by varying different sensing and reporting channel parameters encapsulated in the non-linear forms (48) and (54). For all the experiments, $P_{f}=10^{-2}, N=60$.

Experiment 3: Figs. 4, 5 and 6 show $P_{\text {det }}$ vs the ratio $\sigma_{1}^{2} / \sigma_{0}^{2}$ between the noise variances of $\mathrm{SU}_{0}$ and $\mathrm{SU}_{1}$ on the $\mathrm{SCs}$, for all the considered cooperative sensing techniques and for different values of $L_{e}$, when $M=3$. The results are obtained by fixing $\mathrm{SNR}_{0}=-8 \mathrm{~dB}$, i.e., by fixing $\sigma_{0}^{2}$, and letting $\sigma_{1}^{2}$ to vary. The SNR and the noise variances are set as follows: $\mathrm{SNR}_{2}=-8.6 \mathrm{~dB}, \mathrm{SNR}_{3}=-9.2 \mathrm{~dB}, \delta_{1}^{2}=0.8, \delta_{2}^{2}=0.2$ and $\delta_{3}^{2}=0.4$. In the figures, the red squares denote the points in which the two decision approaches perform the same, i.e, the threshold values in terms of $\sigma_{1}^{2} / \sigma_{0}^{2}$. The results confirm the presence of the threshold behavior for the detection accuracy, regardless of the considered cooperative sensing technique. In particular, for all the considered techniques, when $L_{e}$ increases, the threshold value in terms of $\sigma_{1}^{2} / \sigma_{0}^{2}$ decreases. This agrees with the intuition, in fact, when $L_{e}$ increases, the SCD approach benefits from processing an increased number of local sensing statistics not affected by the RC impairments. Hence, for smaller values of $\sigma_{1}^{2} / \sigma_{0}^{2}$, the SCD is able to outperform the CD. Moreover, it is confirmed the validity of Remark 11 for all the considered cooperative techniques.

Experiment 4: Figs. 7, 8 and 9 show $P_{\text {det }}$ vs $\sigma_{1}^{2} / \sigma_{0}^{2}$, for different values of $M$, when $L_{e}=2, \mathrm{SNR}_{4}=-10 \mathrm{~dB}$, $\delta_{4}^{2}=0.6$, and the remaining parameters are set according to
Experiment 3. For all the cooperative techniques, the results show that when the number $M$ of cooperative SUs increases, the threshold value in terms of $\sigma_{1}^{2} / \sigma_{0}^{2}$ does not change. This agrees with the analytical results (Corollary 1 and 3), assuring that cooperative $\mathrm{SUs}$ different from $\mathrm{SU}_{0}$ and $\mathrm{SU}_{1}$ affect the performance of both the decision approaches in the same way.

Experiment 5: Figs. 10, 11 and 12 show $P_{\text {det }}$ vs $\delta_{1}^{2}$, i.e., vs the $\mathrm{SU}_{1}$ noise variance at $\mathrm{SU}_{0}$ side, for all the considered cooperative sensing techniques and for different values of $L_{g_{1}}$, when $M=3$ and $M=4, L_{e}=1$, and $\sigma_{1}^{2} / \sigma_{0}^{2}=1$. The SNRs are set according to Experiment 2 and the noise variances of the remain SUs are set equal to $\delta_{2}^{2}=0.2, \delta_{3}^{2}=0.4$ and $\delta_{4}^{2}=0.6$. Clearly, the performances of the cooperative techniques implemented by adopting the SCD approach do not depend on $L_{g_{1}}$ and $\delta_{1}^{2}$, as confirmed by the performance curves parallel to the abscissa axis. The results show that when $L_{g_{1}}$ increases, the threshold value in terms of $\delta_{1}^{2}$ increases as well, in agreement with Remark 10 and Corollary 3 for all the considered cooperative techniques. This means that when $L_{g_{1}}$ increases, the CD approach assures higher detection accuracy than the SCD one for a wider range of $\delta_{1}^{2}$ values. It is confirmed that the multipath fading on the $\mathrm{RC}$ introduces degrees of freedom that the cooperative strategies implemented according to the CD approach are able to exploit.

Experiment 6: Finally, Figs. 13, 14 and 15 show $P_{\text {det }}$ vs $\sigma_{1}^{2} / \sigma_{0}^{2}$, when $L_{g_{1}} \rightarrow 1$, i.e., when the RC between $\mathrm{SU}_{0}$ and $\mathrm{SU}_{1}$ is not temporal dispersive, for different values of the $\mathrm{SU}_{1}$ noise power $\delta_{1}^{2}$ at the $\mathrm{SU}_{0}$ side, $M=3$ and $L_{e}=1$ (the SNRs and $\left\{\delta_{i}\right\}_{i=2}^{3}$ are set as in the previous experiment). The results confirm again the presence of the threshold behavior for the detection accuracy, regardless of the considered cooperative sensing technique. Moreover, when $\delta_{1}^{2}$ increases, the threshold value in terms of $\sigma_{1}^{2} / \sigma_{0}^{2}$ decreases, as expected. This means that the SCD approach outperforms the CD one for smaller value of $\sigma_{1}^{2} / \sigma_{0}^{2}$. In fact, when $\delta_{1}^{2}$ increases, the propagation conditions on the $\mathrm{RC}$ between $\mathrm{SU}_{1}$ and $\mathrm{SU}_{0}$ worsen, and therefore, it is enough a small degradation of the $\mathrm{SU}_{1}$ sensing conditions (increased $\sigma_{1}^{2}$ ) to have a significant performance loss by adopting the CD approach with respect to the SCD one (Remark 12).

\section{CONCLUSIONS}

Cooperative spectrum sensing techniques are mainly based on two different decision approaches, according to the role 
of the decision maker: the Combining Decision approach and the Sensing \& Combining Decision approach. The choice of the decision approach deeply affects the performance of any cooperative spectrum sensing technique. Thus, in this paper, by accounting for the effects of realistic channel propagation, a theoretical analysis has been developed to single out the criteria for choosing the decision approach that maximizes the detection accuracy of an arbitrary cooperative technique, regardless of the underlying cooperative sensing architecture. Specifically, it has been proved that the detection accuracy exhibits a threshold behavior as a function of the adopted decision approach, i.e., it exists a threshold value depending on the sensing and reporting channel parameters that determines two different operating regions. In one region, the higher detection accuracy is assured by implementing the cooperative sensing technique according to the Combining Decision approach, whereas in the other region, the higher detection accuracy is guaranteed by the Sensing \& Combining Decision approach, regardless of the underlying cooperative sensing architecture. Closed-form expressions of the threshold are analytically derived, and practical insights for the decision approach selection have been provided.

\section{APPENDIX A}

\section{PROOF OF PROPOSITION 1}

By exploiting (25), $\mathbf{C}_{\mathbf{y}_{\mathrm{sc}, \mathrm{a}}} \mathcal{H}_{j}$ results rank-deficient, since $2 L_{e}$ rows (equivalently $2 L_{e}$ columns) are equal. In fact, the first $L_{e}$ rows ( $L_{e}$ columns) of both $\mathbf{C}_{\mathbf{y}_{\mathrm{sc}} \mid \mathcal{H}_{j}}$ in (10) and $\mathbf{C}_{\mathbf{y}_{\mathrm{sc}}^{*} \mid \mathcal{H}_{j}}$ in (13) are equal to the real matrix $\mathbf{C}_{\mathbf{y}_{0} \mid \mathcal{H}_{j}}$ in (11). By accounting for footnote 8 , after some algebraic manipulations, it is easy to show that $\mathbf{C}_{\mathbf{y}_{\mathrm{sc}, \mathrm{a}}, \mathcal{H}_{j}}$ admits the full-rank factorization [11]: $\mathbf{C}_{\mathbf{y}_{\text {sc,a }} \mid \mathcal{H}_{j}}=\mathbf{F} \mathbf{\Psi}$, i.e., it can be rewritten as product of a full-column rank matrix $\mathbf{F} \in \mathbb{C}^{\left(2 M L_{e}\right) \times(2 M-1) L_{e}}$ and a full-row rank matrix $\Psi \in \mathbb{R}^{(2 M-1) L_{e} \times 2 M L_{e}}$, given by, respectively, (56) and (57), reported at the top of the next page.

$$
\mathbf{F} \triangleq\left[\begin{array}{ccc}
\mathbf{0}_{L_{e} \times(M-1) L_{e}} & \mathbf{C}_{\mathbf{y}_{0} \mid \mathcal{H}_{j}} & \mathbf{0}_{L_{e} \times(M-1) L_{e}} \\
\mathbf{C}_{\overline{\mathbf{y}} \mid \mathcal{H}_{j}} & \mathbf{0}_{(M-1) L_{e} \times L_{e}} & \mathbf{C}_{\overline{\mathbf{y}}^{*} \mid \mathcal{H}_{j}} \\
\mathbf{0}_{L_{e} \times(M-1) L_{e}} & \mathbf{C}_{\mathbf{y}_{0} \mid \mathcal{H}_{j}} & \mathbf{0}_{L_{e} \times(M-1) L_{e}} \\
\mathbf{C}_{\overline{\mathbf{y}}^{*} \mid \mathcal{H}_{j}}^{*} & \mathbf{0}_{(M-1) L_{e} \times L_{e}} & \mathbf{C}_{\overline{\mathbf{y}} \mid \mathcal{H}_{j}}^{*}
\end{array}\right]
$$

Hence, the Moore-Penrose inverse of $\mathbf{C}_{\mathbf{y}_{\text {sca. }} \mid \mathcal{H}_{j}}$ is equal [11] to: $\mathbf{C}_{\mathbf{y}_{\text {sca }}^{\dagger} \mid \mathcal{H}_{j}}^{\dagger}=(\mathbf{F} \boldsymbol{\Psi})^{\dagger}=\boldsymbol{\Psi}^{H}\left(\boldsymbol{\Psi} \boldsymbol{\Psi}^{H}\right)^{-1}\left(\mathbf{F}^{H} \mathbf{F}\right)^{-1} \mathbf{F}^{H}$. Since $\operatorname{rank}\left(\mathbf{C}_{\mathbf{y}_{\mathrm{sc}, \mathrm{a}} \mid \mathcal{H}_{j}}\right)=\operatorname{rank}\left(\mathbf{C}_{\mathbf{y}_{\mathrm{scca}} \mid \mathcal{H}_{j}}^{\dagger} \mathbf{C}_{\mathbf{y}_{\mathrm{scc},} \mid \mathcal{H}_{j}}\right)=$ $\operatorname{trace}\left(\mathbf{C}_{\mathbf{y}_{\mathrm{sc}, \mathrm{a}} \mid \mathcal{H}_{j}}^{\dagger} \mathbf{C}_{\mathbf{y}_{\mathrm{sc}, \mathrm{a}} \mid \mathcal{H}_{j}}\right)$, being $\mathbf{C}_{\mathbf{y}_{\mathrm{sc}, a} \mid \mathcal{H}_{j}}^{\dagger} \mathbf{C}_{\mathbf{y}_{\mathrm{sc}, \mathrm{a}} \mid \mathcal{H}_{j}}$ idempotent [11], it results: $\operatorname{rank}\left(\mathbf{C}_{\mathbf{y}_{\text {sc. }} \mid \mathcal{H}_{j}}\right)=\operatorname{trace}\left(\left(\boldsymbol{\Psi} \Psi^{H}\right)^{-1} \boldsymbol{\Psi} \Psi^{H}\right)=$ $\operatorname{trace}\left(\mathbf{I}_{(2 M-1) L_{e}}\right)=(2 M-1) L_{e}$.

\section{APPENDIX B}

\section{PROOF OF PROPOSITION 2}

Since $\mathcal{R}\left(\mathbf{C}_{\mathbf{y}_{\mathrm{sc}, \mathrm{a}} \mid \mathcal{H}_{1}}\right)=\mathcal{R}\left(\mathbf{C}_{\mathbf{y}_{\mathrm{sc}, \mathrm{a}} \mid \mathcal{H}_{1}}^{H}\right)$ and $\mathcal{N}\left(\mathbf{C}_{\mathbf{y}_{\mathrm{scc}, \mathrm{a}} \mid \mathcal{H}_{1}}\right)$ are complementary orthogonal subspaces [11], any vector $\mathbf{f}_{\mathrm{sc}} \in$

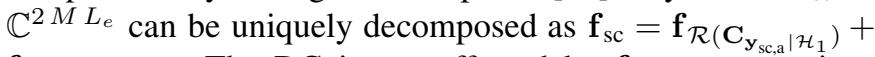

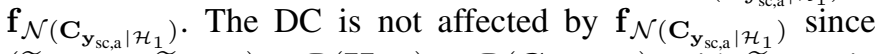
$\left(\widetilde{\boldsymbol{\mu}}_{\mathrm{sc} \mid \mathcal{H}_{1}}-\widetilde{\boldsymbol{\mu}}_{\mathrm{sc} \mid \mathcal{H}_{0}}\right) \in \mathcal{R}\left(\mathbf{H}_{\mathrm{sc}, \mathrm{a}}\right) \subseteq \mathcal{R}\left(\mathbf{C}_{\mathbf{y}_{\mathrm{sc}, \mathrm{a}} \mid \mathcal{H}_{1}}\right)$, with $\widetilde{\boldsymbol{\mu}}_{\mathrm{sc} \mid \mathcal{H}_{j}}$ in (24). Hence, the processing vector $\mathbf{f}_{\mathrm{wl}-\mathrm{sc}}$ maximizing the DC can be equivalently obtained by solving the problem (30).

\section{APPENDIX C \\ ProOF OF THEOREM 1}

Since the solution of (30) belongs to $\mathcal{R}\left(\mathbf{C}_{\mathbf{y}_{\text {sca }} \mid \mathcal{H}_{1}}\right)$ (Propo-

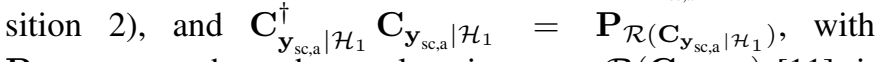
$\mathbf{P}_{\mathcal{R}\left(\mathbf{C}_{\mathbf{y}_{\mathrm{sca}, \mid} \mid \mathcal{H}_{1}}\right)}$ the orthogonal projector on $\mathcal{R}\left(\mathbf{C}_{\mathbf{y}_{\mathrm{a}} \mid \mathcal{H}_{1}}\right)$ [11], it results:

$$
\mathbf{C}_{\mathbf{y}_{\mathrm{sc}, \mathrm{a}} \mid \mathcal{H}_{1}}^{\dagger} \mathbf{C}_{\mathbf{y}_{\mathrm{sc}, \mathrm{a}} \mid \mathcal{H}_{1}} \mathbf{f}_{\mathrm{sc}}=\mathbf{f}_{\mathrm{sc}} \Leftrightarrow \mathbf{f}_{\mathrm{sc}} \in \mathcal{R}\left(\mathbf{C}_{\mathbf{y}_{\mathrm{sc}, \mathrm{a}} \mid \mathcal{H}_{1}}\right)
$$

Hence, by using (58) and the Cauchy-Schwartz's inequality in the DC expression one obtains (59), reported at the top of the next page, where the equality holds if $\mathbf{C}_{\mathbf{y}_{\mathrm{sc}, a} \mid \mathcal{H}_{1}}^{1 / 2} \mathbf{f}_{\mathrm{sc}}=$ $\xi \mathbf{C}_{\mathbf{y}_{\mathrm{sc}, a} \mid \mathcal{H}_{1}}^{1 / 2} \mathbf{C}_{\mathbf{y}_{\mathrm{sc}, a} \mid \mathcal{H}_{1}}^{\dagger}\left(\widetilde{\boldsymbol{\mu}}_{\mathrm{sc} \mid \mathcal{H}_{1}}-\widetilde{\boldsymbol{\mu}}_{\mathrm{sc} \mid \mathcal{H}_{0}}\right) \Leftrightarrow \mathbf{f}_{\mathrm{sc}}=\mathbf{f}_{\mathrm{wl}-\mathrm{sc}}=$ $\xi \mathbf{C}_{\mathbf{y}_{\mathrm{sc}, a} \mid \mathcal{H}_{1}}^{\dagger}\left(\widetilde{\boldsymbol{\mu}}_{\mathrm{sc} \mid \mathcal{H}_{1}}-\widetilde{\boldsymbol{\mu}}_{\mathrm{sc} \mid \mathcal{H}_{0}}\right)$. The constraint $\mathbf{f}_{\mathrm{sc}}^{H} \mathbf{f}_{\mathrm{sc}}=1$ is satisfied by $\xi=1 /\left\|\mathbf{C}_{\mathbf{y}_{\text {sca }} \mid \mathcal{H}_{1}}^{\dagger}\left(\widetilde{\boldsymbol{\mu}}_{\mathrm{sc} \mid \mathcal{H}_{1}}-\widetilde{\boldsymbol{\mu}}_{\mathrm{sc} \mid \mathcal{H}_{0}}\right)\right\|_{2}^{2}$, which is real to assure the conjugate symmetric property of $\mathbf{f}_{\mathrm{sc}}$.

\section{APPENDIX D \\ PROOF OF PROPOSITION 3}

Sensing \& Combining Decision approach case: For Proposition $1, \mathbf{C}_{\mathbf{y}_{\text {sca. }} \mid \mathcal{H}_{1}}$ has at least one non-singular submatrix $\mathbf{X}_{11} \in \mathbb{C}^{(2 M-1) L_{e} \times(2 M-1) L_{e}}$ such that: $\mathbf{P C}_{\mathbf{y}_{\mathrm{sc}, \mathrm{a}} \mid \mathcal{H}_{1}} \mathbf{Q}=$ $\left[\begin{array}{ll}\mathbf{X}_{11} & \mathbf{X}_{12} \\ \mathbf{X}_{21} & \mathbf{X}_{22}\end{array}\right]$, where $\mathbf{P}$ and $\mathbf{Q}$ are permutation matrices, [11]. By defining $\mathbf{P} \triangleq\left[\begin{array}{cc}\mathbf{0}_{(2 M-1) L_{e} \times L_{e}} & \mathbf{I}_{(2 M-1) L_{e}} \\ \mathbf{I}_{L_{e}} & \mathbf{0}_{L_{e} \times(2 M-1) L_{e}}\end{array}\right] \in$ $\mathbb{R}^{2 M L_{e} \times 2 M L_{e}}$ and $\mathbf{Q} \triangleq \mathbf{P}^{T}$, it results:

$$
\begin{aligned}
\mathbf{X}_{11}= & {\left[\begin{array}{cc}
{\left[\begin{array}{cc}
\mathbf{C}_{\overline{\mathbf{y}} \mid \mathcal{H}_{1}} & \mathbf{0}_{(M-1) L_{e} \times L_{e}} \\
\mathbf{0}_{L_{e} \times(M-1) L_{e}} & \mathbf{C}_{\mathbf{y}_{0} \mid \mathcal{H}_{1}} \\
{\left[\mathbf{C}_{\overline{\mathbf{y}}^{*} \mid \mathcal{H}_{1}}^{*}\right.} & \mathbf{0}_{(M-1) L_{e} \times L_{e}}^{*}
\end{array}\right]} & {\left[\begin{array}{c}
\mathbf{C}_{\overline{\mathbf{y}}^{*} \mid \mathcal{H}_{1}} \\
\mathbf{0}_{L_{e} \times(M-1) L_{e}}
\end{array}\right]} \\
{\left[\mathbf{C}_{\overline{\mathbf{y}} \mid \mathcal{H}_{1}}^{*}\right.}
\end{array}\right] } \\
\mathbf{X}_{21} & =\left[\begin{array}{lll}
\mathbf{0}_{L_{e} \times(M-1) L_{e}} & \mathbf{C}_{\mathbf{y}_{0} \mid \mathcal{H}_{1}} & \mathbf{0}_{L_{e} \times(M-1) L_{e}}
\end{array}\right]=\mathbf{X}_{12}^{T} \\
\mathbf{X}_{22} & =\mathbf{C}_{\mathbf{y}_{0} \mid \mathcal{H}_{1}} .
\end{aligned}
$$

By substituting (60) and (61) in the Noble's equality [11], and by denoting with $\mathbf{C}_{\mathbf{y}_{\mathrm{sc}, \mathrm{a}} \mid \mathcal{H}_{1}}^{\dagger} \triangleq\left[\begin{array}{ll}\boldsymbol{\Phi}_{11} & \boldsymbol{\Phi}_{12} \\ \boldsymbol{\Phi}_{21} & \mathbf{\Phi}_{22}\end{array}\right]$, after some algebraic manipulations, we obtain: $\boldsymbol{\Phi}_{11}=$ $\boldsymbol{\Phi}_{22}^{*}=\left[\begin{array}{cc}\frac{1}{4} \mathbf{C}_{\mathbf{y}_{0} \mid \mathcal{H}_{1}}^{-1} & \mathbf{0}_{L_{e} \times(M-1) L_{e}} \\ \mathbf{0}_{(M-1) L_{e} \times L_{e}} & \overline{\boldsymbol{\Omega}}\end{array}\right], \boldsymbol{\Phi}_{12}=\boldsymbol{\Phi}_{21}^{*}=$ $\left[\begin{array}{cc}\frac{1}{4} \mathbf{C}_{\mathbf{y}_{0} \mid \mathcal{H}_{1}}^{-1} & \mathbf{0}_{L_{e} \times(M-1) L_{e}} \\ \mathbf{0}_{(M-1) L_{e} \times L_{e}} & -\mathbf{C}_{\overline{\mathbf{y}} \mid \mathcal{H}_{1}}^{-1} \mathbf{C}_{\overline{\mathbf{y}}^{*} \mid \mathcal{H}_{1}} \overline{\boldsymbol{\Omega}}^{*}\end{array}\right]$, with $\overline{\boldsymbol{\Omega}}$ given in (44). By exploiting (31), the WL subvector $\mathbf{t}_{\mathrm{wl}-\mathrm{sc}}$ is expressed as:

$$
\mathbf{t}_{\mathrm{wl}-\mathrm{sc}}=\frac{\left(\boldsymbol{\Phi}_{11} \mathbf{H}_{\mathrm{sc}}+\boldsymbol{\Phi}_{12} \mathbf{H}_{\mathrm{sc}}^{*}\right)\left(\boldsymbol{\eta}_{\mathrm{sc} \mid \mathcal{H}_{1}}-\boldsymbol{\eta}_{\mathrm{sc} \mid \mathcal{H}_{0}}\right)}{\sqrt{2}\left\|\left(\boldsymbol{\Phi}_{11} \mathbf{H}_{\mathrm{sc}}+\boldsymbol{\Phi}_{12} \mathbf{H}_{\mathrm{sc}}^{*}\right)\left(\boldsymbol{\eta}_{\mathrm{sc} \mid \mathcal{H}_{1}}-\boldsymbol{\eta}_{\mathrm{sc} \mid \mathcal{H}_{0}}\right)\right\|_{2}^{2}}
$$

The proof is achieved by substituting $\boldsymbol{\Phi}_{11}$ and $\boldsymbol{\Phi}_{12}$ in (62) and by using the expression of $\mathbf{H}_{\mathrm{sc}}$.

Combining Decision approach case: By exploiting (28), and by denoting with $\mathbf{C}_{\mathbf{y}_{c, a} \mid \mathcal{H}_{1}}^{-1} \triangleq\left[\begin{array}{ll}\mathbf{A}_{11} & \mathbf{A}_{12} \\ \mathbf{A}_{21} & \mathbf{A}_{22}\end{array}\right]$, the WL subvector $\mathbf{t}_{\mathrm{wl}-\mathrm{c}}$ can be expressed as:

$$
\mathbf{t}_{\mathrm{wl}-\mathrm{c}}=\frac{\left[\mathbf{A}_{11} \mathbf{H}_{c}+\mathbf{A}_{12} \mathbf{H}_{c}^{*}\right]\left(\boldsymbol{\eta}_{\mathrm{c} \mid \mathcal{H}_{1}}-\boldsymbol{\eta}_{\mathrm{c} \mid \mathcal{H}_{0}}\right)}{\sqrt{2} \|\left.\left[\mathbf{A}_{11} \mathbf{H}_{c}+\mathbf{A}_{12} \mathbf{H}_{c}^{*}\right]\left(\boldsymbol{\eta}_{\mathrm{c} \mid \mathcal{H}_{1}}-\boldsymbol{\eta}_{\mathrm{c} \mid \mathcal{H}_{0}}\right)\right|_{2} ^{2}}
$$




$$
\begin{aligned}
& \boldsymbol{\Psi} \triangleq\left[\begin{array}{cccc}
\mathbf{0}_{(M-1) L_{e} \times L_{e}} & \mathbf{I}_{(M-1) L_{e}} & \mathbf{0}_{(M-1) L_{e} \times L_{e}} & \mathbf{0}_{(M-1) L_{e} \times(M-1) L_{e}} \\
\mathbf{I}_{L_{e}} & \mathbf{0}_{L_{e} \times(M-1) L_{e}} & \mathbf{I}_{L_{e}} & \mathbf{0}_{L_{e} \times(M-1) L_{e}} \\
\mathbf{0}_{(M-1) L_{e} \times L_{e}} & \mathbf{0}_{(M-1) L_{e} \times(M-1) L_{e}} & \mathbf{0}_{(M-1) L_{e} \times L_{e}} & \mathbf{I}_{(M-1) L_{e}}
\end{array}\right] \\
& m_{\mathrm{wl}}^{2}\left(\mathbf{f}_{\mathrm{sc}}\right)=\frac{\left|\left(\mathbf{C}_{\mathbf{y}_{\mathrm{sc}, a} \mid \mathcal{H}}^{1 / 2} \mathbf{f}_{\mathrm{sc}}\right)^{H} \mathbf{C}_{\mathbf{y}_{\mathrm{sc}, a} \mid \mathcal{H}_{1}}^{1 / 2} \mathbf{C}_{\mathbf{y}_{\mathrm{sc}, a} \mid \mathcal{H}_{1}}^{\dagger}\left(\widetilde{\boldsymbol{\mu}}_{\mathrm{sc} \mid \mathcal{H}_{1}}-\widetilde{\boldsymbol{\mu}}_{\mathrm{sc} \mid \mathcal{H}_{0}}\right)\right|^{2}}{\mathbf{f}_{\mathrm{sc}}^{H} \mathbf{C}_{\mathbf{y}_{\mathrm{sc}, \mathrm{a}} \mid \mathcal{H}_{1}} \mathbf{f}_{\mathrm{sc}}} \leq\left(\widetilde{\boldsymbol{\mu}}_{\mathrm{sc} \mid \mathcal{H}_{1}}-\widetilde{\boldsymbol{\mu}}_{\mathrm{sc} \mid \mathcal{H}_{0}}\right)^{H} \mathbf{C}_{\mathbf{y}_{\mathrm{sc}, \mathrm{a}} \mid \mathcal{H}_{1}}^{\dagger}\left(\widetilde{\boldsymbol{\mu}}_{\mathrm{sc} \mid \mathcal{H}_{1}}-\widetilde{\boldsymbol{\mu}}_{\mathrm{sc} \mid \mathcal{H}_{0}}\right)
\end{aligned}
$$

By accounting for the inverse of a partitioned matrix [17], it results: $\mathbf{A}_{11}=\left[\mathbf{C}_{\mathbf{y}_{c} \mid \mathcal{H}_{1}}-\mathbf{C}_{\mathbf{y}_{c}^{*} \mid \mathcal{H}_{1}}\left(\mathbf{C}_{\mathbf{y}_{c} \mid \mathcal{H}_{1}}^{*}\right)^{-1} \mathbf{C}_{\mathbf{y}_{c}^{*} \mid \mathcal{H}_{1}}^{*}\right]^{-1}$ and $\mathbf{A}_{12}=-\mathbf{C}_{\mathbf{y}_{c} \mid \mathcal{H}_{1}}^{-1} \mathbf{C}_{\mathbf{y}_{c}^{*} \mid \mathcal{H}_{1}} \mathbf{A}_{11}^{*}$. By substituting (18), (20), $\mathbf{A}_{11}$, $\mathbf{A}_{12}$ in (63), the proof is achieved.

\section{APPENDIX E \\ PROOF OF THEOREM 2}

The proof is carried out by comparing the WL deflection coefficients (29) and (32) assured by the CD and the SCD approach, respectively, since, under the gaussian hypothesis, a larger deflection coefficient corresponds to a major detection accuracy [4], [9], [14]. To this aim, (29) and (32) can be rewritten, respectively, as: $m_{\mathrm{wl-c}}^{2}\left(\mathbf{f}_{\mathrm{wl-c}}\right)=$ $2 \sqrt{2}\left\|\boldsymbol{\Sigma}_{\mathrm{wl}-\mathrm{c}}\left(\boldsymbol{\eta}_{\mathrm{c} \mid \mathcal{H}_{1}}-\boldsymbol{\eta}_{\mathrm{c} \mid \mathcal{H}_{0}}\right)\right\|_{2}^{2} \operatorname{Re}\left[\left(\boldsymbol{\mu}_{\mathrm{c} \mid \mathcal{H}_{1}}-\boldsymbol{\mu}_{\mathrm{c} \mid \mathcal{H}_{0}}\right)^{H} \mathbf{t}_{\mathrm{wl-c}}\right]$ and $\quad m_{\mathrm{wl}-\mathrm{sc}}^{2}\left(\mathbf{f}_{\mathrm{wl}-\mathrm{sc}}\right)=2 \sqrt{2} \| \boldsymbol{\Sigma}_{\mathrm{wl}-\mathrm{sc}}\left(\boldsymbol{\eta}_{\mathrm{sc} \mid \mathcal{H}_{1}}-\right.$ $\left.\boldsymbol{\eta}_{\mathrm{sc} \mid \mathcal{H}_{0}}\right) \|_{2}^{2} \operatorname{Re}\left[\left(\boldsymbol{\mu}_{\mathrm{sc} \mid \mathcal{H}_{1}}-\boldsymbol{\mu}_{\mathrm{sc} \mid \mathcal{H}_{0}}\right)^{H} \mathbf{t}_{\mathrm{wl}-\mathrm{sc}}\right] . \quad$ By substituting (45) in these expressions, after some manipulations, one obtains (64) and (65), reported at the top of the next page, where, the positive common term, i.e., $2 \operatorname{Re}\left\{\left(\overline{\boldsymbol{\eta}}_{\mathcal{H}_{1}}-\right.\right.$ $\left.\left.\overline{\boldsymbol{\eta}}_{\mathcal{H}_{0}}\right)^{T} \overline{\mathbf{H}}^{H}\left(\overline{\mathbf{\Omega}}-\mathbf{C}_{\overline{\mathbf{y}} \mid \mathcal{H}_{1}}^{-1} \mathbf{C}_{\overline{\mathbf{y}}^{*} \mid \mathcal{H}_{1}} \overline{\mathbf{\Omega}}^{*} \overline{\mathbf{H}}^{*}\right)\left(\overline{\boldsymbol{\eta}}_{\mathcal{H}_{1}}-\overline{\boldsymbol{\eta}}_{\mathcal{H}_{0}}\right)\right\}$, depends on the $(M-1)$ SUs different from $\mathrm{SU}_{0}$ and $\mathrm{SU}_{1}$. Consequently,

$$
\begin{aligned}
& m_{\mathrm{wl-sc}}^{2} \gtreqless m_{\mathrm{wl-c}}^{2} \Leftrightarrow \\
& \left(\nu_{0 \mid \mathcal{H}_{1}}-\nu_{0 \mid \mathcal{H}_{0}}\right)^{2} \zeta_{0 \mid \mathcal{H}_{1}}^{-1} L_{e} \gtreqless 2\left(\nu_{1 \mid \mathcal{H}_{1}}-\nu_{1 \mid \mathcal{H}_{0}}\right)^{2} \mathbf{1}_{\left\{L_{e}+L_{g}-1\right\}}^{T} \\
& \operatorname{Re}\left[\mathbf{H}_{1}^{H}\left(\boldsymbol{\Omega}_{\mathrm{c}} \mathbf{H}_{1}-\mathbf{C}_{\mathbf{y}_{1} \mid \mathcal{H}_{1}}^{-1} \mathbf{C}_{\mathbf{y}_{1}^{*} \mid \mathcal{H}_{1}} \boldsymbol{\Omega}_{\mathrm{c}}^{*} \mathbf{H}_{1}^{*}\right)\right] \mathbf{1}_{\left\{L_{e}+L_{g}-1\right\}}
\end{aligned}
$$

where both the members are positive, due to the DC definition. From (66), it results (67), reported at the top of the next page. Hence, by denoting with $\theta_{\mathrm{wl}}$ the positive quantity defined in (48), (67) is equivalent to $m_{\text {wl-sc }}^{2} \gtreqless m_{\text {wl-c }}^{2} \Leftrightarrow \theta_{\text {wl }} \gtreqless 1$.

\section{REFERENCES}

[1] S. Haykin, "Cognitive radio: brain-empowered wireless communications," IEEE J. Sel. Areas Commun., vol. 23, no. 2, pp. 201-220, Feb. 2005.

[2] A. S. Cacciapuoti, I. F. Akyildiz, and L. Paura, "Correlation-aware user selection for cooperative spectrum sensing in cognitive radio ad hoc networks," IEEE J. Sel. Areas Commun., vol. 30, no. 2, pp. 297-306, Feb. 2012 .

[3] I. F. Akyildiz and B. F. Lo, and R. Balakrishnan, "Cooperative spectrum sensing in cognitive radio networks: a survey," Physical Commun. J., vol. 4, no. 1, Mar. 2011.

[4] A. S. Cacciapuoti, M. Caleffi, D. Izzo, and L. Paura, "Cooperative spectrum sensing techniques with temporal dispersive reporting channels," IEEE Trans. Wireless Commun., vol. 10, no. 10, pp. 3392-3402, Oct. 2011.

[5] S. Mishra, A. Sahai, and R. Brodersen, "Cooperative sensing among cognitive radios," in Proc. 2009 IEEE ICC.
[6] A. Ghasemi and E. Sousa, "Collaborative spectrum sensing for opportunistic access in fading environments," in Proc. 2005 IEEE DySPAN.

[7] K. Ben Letaief and W. Zhang, "Cooperative communications for cognitive radio networks," Proc. IEEE, vol. 97, no. 5, May 2009.

[8] I. F. Akyildiz, W.-Y. Lee, and K. R. Chowdhury, "Crahns: cognitive radio ad hoc networks," Ad Hoc Networks, vol. 7, no. 5, pp. 810-836, 2009.

[9] S. M. Kay, Fundamentals of Statistical Signal Processing II: Detection Theory. Prentice-Hall, 1998.

[10] A. S. Cacciapuoti, M. Caleffi, and L. Paura, "Widely linear cooperative spectrum sensing for cognitive radio networks," in Proc. 2010 IEEE GLOBECOM, pp. $1-5$.

[11] A. Ben-Israel and T. N. E. Greville, Generalized Inverses: Theory and Applications. Springer-Verlag, 2002.

[12] P. Schreier and L. Scharf, "Second-order analysis of improper complex random vectors and processes," IEEE Trans. Signal Process., vol. 51, no. 3, pp. 714-725, Mar. 2003

[13] Y.-C. Liang, Y. Zeng, E. C. Peh, and A. T. Hoang, "Sensing-throughput tradeoff for cognitive radio networks," IEEE Trans. Wireless Commun., vol. 7, no. 4, pp. 1326-1337, Apr. 2008.

[14] Z. Quan, S. Cui, and A. H. Sayed, "Optimal linear cooperation for spectrum sensing in cognitive radio networks," IEEE J. Sel. Topics Signal Process., vol. 2, no. 1, Feb 2008.

[15] B. Picinbono, "Second-order complex random vectors and normal distributions," IEEE Trans. Signal Process., vol. 44, no. 10, pp. $2637-$ 2640, Oct. 1996.

[16] P. V. Mikheev, "Multi-dimensional Gaussian probability density and its applications in the degenerate case," Radiophysics and Quantum Electron., vol. 49, no. 7, pp. 626-634, July 2006.

[17] R. A. Horn and C. R. Johnson, Matrix Analysis. Cambridge University Press, 1990.

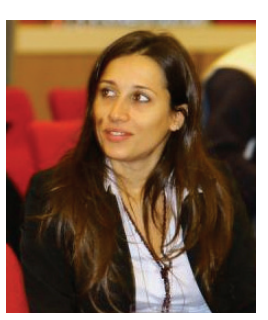

Angela Sara Cacciapuoti received the Dr. Eng. degree summa cum laude in Telecommunications Engineering in 2005, and the Ph.D degree with score "excellent in Electronic and Telecommunications Engineering in 2009, both from University of Naples Federico II. Currently, she is an Assistant Professor with the Department of Electric Engineering and Information Technology (DIETI), University of Naples Federico II. From 2010 to 2011, Dr. Cacciapuoti has been with the Broadband Wireless Networking Laboratory, Georgia Institute of Technology, as visiting researcher. In 2011, she has also been with the NaNoNetworking Center in Catalunya (N3Cat), Universitat Politècnica de Catalunya (UPC), as visiting researcher. Her current research interests are in cognitive radio networks and non-stationary signal processing.

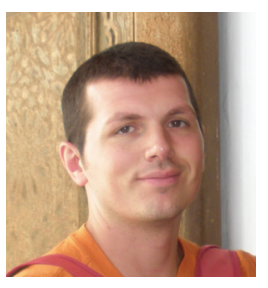

Marcello Caleffi received the Dr. Eng. degree summa cum laude in Computer Science Engineering from University of Lecce in 2005, and the Ph.D. degree in Electronic and Telecommunications Engineering from University of Naples Federico II in 2009. Currently, he is Assistant Professor with the Department of Electrical Engineering and Information Technology (DIETI), University of Naples Federico II. From 2010 to 2011, he was with Broadband Wireless Networking Laboratory, Georgia Institute of Technology, as visiting researcher. In 2011, he was also with NaNoNetworking Center in Catalunya (N3Cat), Universitat Politècnica de Catalunya (UPC), as visiting research. His research interests are in cognitive radio networks and human-enabled wireless networks. 


$$
\begin{gathered}
m_{\mathrm{wl-c}}^{2}\left(\mathbf{f}_{\mathrm{wl-c}}\right)=2\left(\nu_{1 \mid \mathcal{H}_{1}}-\nu_{1 \mid \mathcal{H}_{0}}\right)^{2} \mathbf{1}_{\left\{L_{e}+L_{g}-1\right\}}^{T} \operatorname{Re}\left[\mathbf{H}_{1}^{H}\left(\boldsymbol{\Omega}_{\mathrm{c}} \mathbf{H}_{1}-\mathbf{C}_{\mathbf{y}_{1} \mid \mathcal{H}_{1}}^{-1} \mathbf{C}_{\mathbf{y}_{1}^{*} \mid \mathcal{H}_{1}} \boldsymbol{\Omega}_{\mathrm{c}}^{*} \mathbf{H}_{1}^{*}\right)\right] \\
\mathbf{1}_{\left\{L_{e}+L_{g}-1\right\}}+2 \operatorname{Re}\left\{\left(\overline{\boldsymbol{\eta}}_{\mathcal{H}_{1}}-\overline{\boldsymbol{\eta}}_{\mathcal{H}_{0}}\right)^{T} \overline{\mathbf{H}}^{H}\left(\overline{\boldsymbol{\Omega}} \overline{\mathbf{H}}-\mathbf{C}_{\overline{\mathbf{y}} \mid \mathcal{H}_{1}}^{-1} \mathbf{C}_{\overline{\mathbf{y}}^{*} \mid \mathcal{H}_{1}} \overline{\boldsymbol{\Omega}}^{*} \overline{\mathbf{H}}^{*}\right)\left(\overline{\boldsymbol{\eta}}_{\mathcal{H}_{1}}-\overline{\boldsymbol{\eta}}_{\mathcal{H}_{0}}\right)\right\} \\
m_{\mathrm{wl-sc}}^{2}\left(\mathbf{f}_{\mathrm{wl}-\mathrm{sc}}\right)=\left(\nu_{0 \mid \mathcal{H}_{1}}-\nu_{0 \mid \mathcal{H}_{0}}\right)^{2} \zeta_{0 \mid \mathcal{H}_{1}}^{-1} L_{e}+2 \operatorname{Re}\left\{\left(\overline{\boldsymbol{\eta}}_{\mathcal{H}_{1}}-\overline{\boldsymbol{\eta}}_{\mathcal{H}_{0}}\right)^{T} \overline{\mathbf{H}}^{H}\left(\overline{\boldsymbol{\Omega}} \overline{\mathbf{H}}-\mathbf{C}_{\overline{\mathbf{y}} \mid \mathcal{H}_{1}}^{-1} \mathbf{C}_{\overline{\mathbf{y}}^{*} \mid \mathcal{H}_{1}} \overline{\boldsymbol{\Omega}}^{*} \overline{\mathbf{H}}^{*}\right)\left(\overline{\boldsymbol{\eta}}_{\mathcal{H}_{1}}-\overline{\boldsymbol{\eta}}_{\mathcal{H}_{0}}\right)\right\} \\
m_{\mathrm{wl-sc}}^{2} \gtreqless m_{\mathrm{wl-c}}^{2} \Leftrightarrow \frac{\left(\nu_{0 \mid \mathcal{H}_{1}}-\nu_{0 \mid \mathcal{H}_{0}}\right)^{2} \zeta_{0 \mid \mathcal{H}_{1}}^{-1} L_{e}}{2\left(\nu_{1 \mid \mathcal{H}_{1}}-\nu_{1 \mid \mathcal{H}_{0}}\right)^{2} \mathbf{1}_{\left\{L_{e}+L_{g}-1\right\}}^{T} \operatorname{Re}\left[\mathbf{H}_{1}^{H}\left(\boldsymbol{\Omega}_{\mathrm{c}} \mathbf{H}_{1}-\mathbf{C}_{\mathbf{y}_{1} \mid \mathcal{H}_{1}}^{-1} \mathbf{C}_{\mathbf{y}_{1}^{*} \mid \mathcal{H}_{1}} \boldsymbol{\Omega}_{\mathrm{c}}^{*} \mathbf{H}_{1}^{*}\right)\right] \mathbf{1}_{\left\{L_{e}+L_{g}-1\right\}}} \gtreqless 1
\end{gathered}
$$

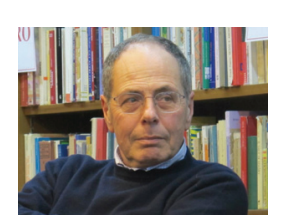

Luigi Paura received the Dr. Eng. degree summa cum laude in Electronic Engineering in 1974 from University of Napoli Federico II. From 1979 to 1984, he was with the Department of Biomedical, Electronic and Telecommunications Engineering, University of Naples Federico II, first as an Assistant Professor and then as an Associate Professor. Since 1994, he has been a Full Professor of Telecom.: first, with the Dept. of Mathematics, University of Lecce, Italy; then, with the Dept. of Information Engineering, Second University of Naples; and, finally, from 1998 he has been with the Dept. of Biomedical, Electronic and Telecom. Engineering, University of Naples Federico II. $\mathrm{He}$ also held teaching positions at University of Salerno, at University of Sannio, and at University Parthenope of Naples. In 1985-86 and 1991 he was a visiting researcher at Signal and Image Processing Lab, University of California, Davis. Currently, he is a full professor with the Department of Electrical Engineering and Information Technology (DIETI), University of Naples Federico II. His research interests are mainly in digital communication systems and communication networks.

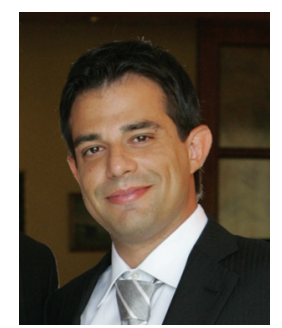

Roberto Savoia received the Dr. Eng. degree summa cum laude in Telecommunications Engineering in 2010 from the University of Napoli Federico II, Italy. Currently, he is pursuing the Ph.D. degree in Electronic and Telecommunications Engineering with the Department of Electric Engineering and Information Technology (DIETI). His research activities lie in the area of wireless communications. In particular, his current interests include cooperative and cognitive radio networks. 